\title{
Space-time Wasserstein controls and Bakry-Ledoux type gradient estimates
}

\author{
Kazumasa Kuwada*
}

October 3, 2018

\begin{abstract}
The duality in Bakry-Émery's gradient estimates and Wasserstein controls for heat distributions is extended to that in refined estimates in a high generality. As a result, we find an equivalent condition to Bakry-Ledoux's refined gradient estimate involving an upper dimension bound. This new condition is described as a $L^{2}$ Wasserstein control for heat distributions at different times. The $L^{p}$-version of those estimates are studied on Riemannian manifolds via coupling method.
\end{abstract}

Key words: gradient estimate, Wasserstein distance, heat distribution, Hopf-Lax semigroup, Ricci curvature

\section{Contents}

1 Introduction 1

2 Framework and main results 5

3 Proof of dualities $\quad 10$

3.1 Reminder of the Hopf-Lax semigroup . . . . . . . . . . . . . . . . 10

3.2 From Wasserstein control to gradient estimates . . . . . . . . . . . . 11

3.3 From gradient estimate to Wasserstein controls . . . . . . . . . . . . 15

4 A coupling method on Riemannian manifolds $\quad 21$

4.1 The case under the absence of the cut locus . . . . . . . . . . . . . 21

4.2 Coupling method via discrete approximation . . . . . . . . . . . . 28

\section{INTRODUCTION}

Since the pioneering work of Bakry and Émery [11], their $\left(L^{2}\right.$-)gradient estimate

$$
\left|\nabla P_{t} f\right|^{2} \leq \mathrm{e}^{-2 K t} P_{t}\left(|\nabla f|^{2}\right)
$$

*Partially supported by the Grant-in-Aid for Young Scientists (B) 22740083 
for a diffusion semigroup $P_{t}=\mathrm{e}^{t \mathscr{L}}$ has been played a prominent role in geometric analysis of the diffusion generator $\mathscr{L}$ (see $[9,10,12,31]$, for instance). Among several further developments, the following refined form is studied by Bakry and Ledoux [15] (see [44] also): For fixed parameters $K \in \mathbb{R}$ and $N \in(0, \infty]$,

$$
\left|\nabla P_{t} f\right|^{2} \leq \mathrm{e}^{-2 K t} P_{t}\left(|\nabla f|^{2}\right)-\frac{1-\mathrm{e}^{-2 K t}}{N K}\left(\mathscr{L} P_{t} f\right)^{2}
$$

Recently, in $[25,28]$, it is revealed that $(1.1)$ is equivalent to the following estimate concerning a Lipschitz type bound of heat distributions with respect to the $L^{2}$-Wasserstein distance $W_{2}$, which we call an $L^{2}$-Wasserstein control, in a fairly general situation: For $t>0$ and two probability measures $\mu_{0}$ and $\mu_{1}$ on the state space,

$$
W_{2}\left(P_{t}^{*} \mu_{0}, P_{t}^{*} \mu_{1}\right) \leq \mathrm{e}^{-K t} W_{2}\left(\mu_{0}, \mu_{1}\right)
$$

The purpose of this article is to extend such a duality by introducing a new inequality like (1.3) which corresponds to (1.2). As we will see in Corollary 2.4 as a special case of our result, the estimate (1.2) is equivalent to the following space-time $L^{2}$-Wasserstein control in an abstract framework: For $s, t>0$ and two probability measures $\mu_{0}$ and $\mu_{1}$ on the state space,

$$
W_{2}\left(P_{s}^{*} \mu_{0}, P_{t}^{*} \mu_{1}\right)^{2} \leq\left(\frac{1}{J_{N}([s, t])} \int_{s}^{t} \mathrm{e}^{K r} J_{N}(d r)\right)^{-2} W_{2}\left(\mu_{0}, \mu_{1}\right)^{2}+J_{N}([s, t])^{2},
$$

where

$$
J_{N}(A):=\int_{A} \sqrt{\frac{N K}{\mathrm{e}^{2 K r}-1}} d r
$$

for measurable $A \subset[0, \infty)$ and hence

$$
J_{N}([s, t])= \begin{cases}\sqrt{\frac{N}{K}}\left(\cos ^{-1}\left(\mathrm{e}^{-K t}\right)-\cos ^{-1}\left(\mathrm{e}^{-K s}\right)\right) & (K>0), \\ \sqrt{2 N}(\sqrt{t}-\sqrt{s}) & (K=0), \\ \sqrt{\frac{N}{-K}}\left(\cosh ^{-1}\left(\mathrm{e}^{-K t}\right)-\cosh ^{-1}\left(\mathrm{e}^{-K s}\right)\right) & (K<0) .\end{cases}
$$

Here the function $\left(\mathrm{e}^{2 K t}-1\right) / K$ is regarded as $2 t$ when $K=0$.

In the Bakry-Ledoux gradient estimate (1.2), the parameters $K$ and $N$ play the role of lower Ricci curvature bound and upper dimension bound. Indeed, under the condition

$$
|\nabla f|^{2}=\frac{1}{2} \mathscr{L}\left(f^{2}\right)-f \mathscr{L} f
$$

the Bakry-Ledoux gradient estimate is equivalent (at least formally) to the following inequality, called Bakry-Émery's curvature-dimension condition or Bochner's inequality

$$
\frac{1}{2} \mathscr{L}\left(|\nabla f|^{2}\right)-\langle\nabla f, \nabla \mathscr{L} f\rangle \geq K|\nabla f|^{2}+\frac{1}{N}|\mathscr{L} f|^{2}
$$


Note that (1.6) is the definition of squared norm of gradient or carré du champ $|\nabla f|^{2}=$ $\langle\nabla f, \nabla f\rangle$ in Bakry-Émery theory, as in [9, 10, 12, 31]. On complete Riemannian manifolds with $\mathscr{L}=\Delta$, the Bochner-Weitzenböck formula implies that Bakry-Émery's curvaturedimension condition is equivalent to the combination of Ric $\geq K$ and $\operatorname{dim} \leq N$ (when $N=\infty$, the latter condition always holds). Moreover, even in an abstract framework, (1.7) has provided several extensions of results in Riemannian geometry concerning these bounds. Thus we could say that (1.1) or (1.2) is placed at the intersection of geometry and analysis. While (1.1) can be applied in a broader situation such as analysis on infinite dimensional spaces, (1.2) provides qualitatively sharper results and hence obtaining (1.2) or (1.7) for $N<\infty$ would be important (see [9, 10, 12, 31] for instance).

The Wasserstein control condition (1.3) or (1.4) serves us a new approach to (1.1) or (1.2) especially on non-smooth spaces. Since the Bochner-Weitzenböck formula is not available in such a case, it was completely unclear when (1.7) holds. For this problem, a connection with an alternative formulation of "Ric $\geq K$ and $\operatorname{dim} \leq N$ " by optimal transportation $[8,33,38,39]$ has been investigated recently. Since those new conditions are stable under geometric operations such as the measured Gromov-Hausdorff limit, the same stability holds for (1.7) once we prove the equivalence between them. This equivalence is finally established by Ambrosio, Gigli, Savaré, Mondino and Rajala [1, 2, 3, 4] when $N=\infty$ and by Erbar, Sturm and the author [20] when $N<\infty$. For connecting BakryÈmery theory based on (1.7) with optimal transport approach, the estimate (1.3) or (1.4) works as a bridge, though what we actually used when $N<\infty$ is (2.8) below.

The emphasis of the result of this paper is put on the fact that the equivalence between (1.2) and (1.4) can be extended to more general situation where any kind of known curvature-dimension conditions corresponding to "Ric $\geq K$ and $\operatorname{dim} \leq N$ " may not hold (see Theorem 2.1). For instance, previous results can be applied to obtain an estimate like (1.3) from an estimate like (1.1) for sub-elliptic diffusions [25, Section 4] (see [28, Section 6] also for other examples). We can expect a similar result also in the present case as a future application. As another kind of generality, we can obtain $L^{p} / L^{p_{*}}$-duality, as we did in $[25,28]$. Actually, an $L^{p}$-type estimate of (1.4) (see (2.10)) holds on complete

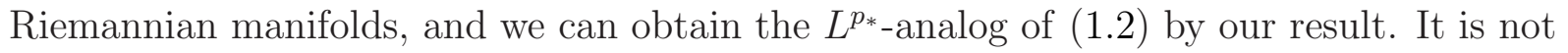
yet known whether we can obtain the same estimate on metric measure spaces satisfying the Riemannian curvature-dimension condition in [20], where the $L^{2}$-estimate holds. Note that, in the case $N=\infty$, the $L^{p}$-version of (1.3) follows from (1.3) itself (see [37]). Such a precision has various applications in that case and hence it would be interesting to obtain an $L^{p}$-estimate associated with the curvature-dimension condition as well as to investigate applications of it.

The essential idea of the proof of the duality in (1.2) and (1.4) is inherited from the earlier studies in $[25,28]$. There we regard (1.1) as a differentiation of (1.3) in space variable with a change of viewpoint from the space of measures to the space of functions. Then the opposite implication is regarded as an integration in space variable, and it is realized by using the Kantorovich duality and the analysis of the associated Hopf-Lax semigroup. Thus it was natural in those argument that we used no time-dependency on the constant and the Markov kernel $P_{t}$. In the present case, the additional term in (1.2) involving $N$ can be regarded as a differentiation in time parameter. The reason why the "integrated" estimate (1.4) deals with distribution of diffusions at different times is 
based on this fact. Actually, if we apply (1.4) when $s=t$ by taking a limit $s \rightarrow t$, it becomes the reduced control (1.3), which is in duality with (1.1). In the proof of our main theorem, we will couple a space parameter with a time parameter. As a result, the argument becomes more complicated compared with previous ones in [25, 28]. This fact also makes it unclear what is the optimal bound of space-time Wasserstein control of type (1.4). Indeed, we obtain (1.4) by choosing a (possibly not optimal but admissible) space-time reparametrizations in a variational problem arising in Proposition 3.5 below (see Remark 3.7). When we are working with the specified estimate (1.2), the space-time $W_{2}$-control (2.8) involving comparison functions seems to be optimal (see Remark 3.8).

$L^{p}$-type estimates on Riemannian manifolds are obtained by stochastic analytic techniques. We construct a variant of coupling by parallel transport of diffusion processes with different time scales for deriving Wasserstein controls. For the construction, we must avoid technical difficulties arising from the presence of the cut locus. To overcome it, we employ the approximation of the diffusion process by geodesic random walks developed in $[24,26,30,42]$. The Wasserstein control we will obtain directly from our coupling method is slightly weaker than expected and we establish an $L^{p}$-variant of Bakry-Émery theory (Proposition 4.4) in order to derive a sharper result. Since we consider a possibly nonsymmetric diffusion generator, we relies on stochastic analytic techniques again to avoid technical difficulties there. It might be possible to prove it in a more straightforward way without coupling methods. See Remark 4.6 for an observation.

As a related result, the implication from (1.2) to (1.4) is obtained by Bakry, Gentil and Ledoux in [13] by a different method when $K=0$. Bolley, Gentil and Guillin [18] recently obtained a sort of contraction bound of $L^{2}$-Wasserstein distance for two distributions of diffusion at the same time involving the dimension parameter $N$ from (1.7). Coupling method on Riemannian manifolds is studied first by Kendall [23] and improved by Cranston [19]. Since then, it has been extensively studied in the literature. Feng-Yu Wang is one of the leading persons on this topic and we refer to his books $[43,45]$ for further developments. A coupling admitting different time scales is studied also in [29, 34]. The problem studied in [29] seems to be closely related to ours (See Remark 2.10). Though the purpose of [34] is different from ours, there appears a similar argument.

The organization of the paper is as follows. In the next section, we give a precise definition of our framework and state the main theorems. Since we will deal with the timeevolution of the Markov kernel, we discuss it under two different family of assumptions in Theorem 2.1 and Theorem 2.2 respectively. In those theorems, only a weaker duality is obtained and we add a technical assumption (strong Feller property of $P_{t}$ ) in both cases to obtain the full duality result including the relation between (1.2) and (1.4) (Corollary 2.3). The proof of main results except Theorem 2.6 is given in Section 3. In the proof, we will show two key propositions: Proposition 3.1 and Proposition 3.5. Though they can be applied to more general situation than in the setting of Theorem 2.1, we exclude it from the main theorem for simplicity of presentation since the statements of them look more complicated. We also prove another space-time $W_{2}$-control (2.8) studied in [20] directly from (1.2) (Theorem 2.5). In Section 4, we will prove Theorem 2.6, which concerns with $L^{p}$-estimates, on a complete Riemannian manifold satisfying (1.7) for a (possibly nonsymmetric) diffusion generator $\mathscr{L}$. Since the argument seems to be technical, we give a 
heuristic discussion in Section 4.1 and make it rigorous in Section 4.2 with a partial use of arguments in Section 4.1 which hold in a sufficient generality.

Acknowledgment: The author would like to tell his gratitude to the anonymous referee. His/Her comments help the author to improve the quality of the paper. Especially, Proposition 4.4 and Remark 4.6 are essentially due to the comment.

\section{FRAMEWORK AND MAIN RESULTS}

Let $(M, d)$ be a Polish metric space. In this paper we always assume that $d$ is a geodesic metric. It means that, for each $x, y \in M$, there is a curve $\gamma:[0,1] \rightarrow M$ such that $\gamma(0)=x, \gamma(1)=y$ and $d(\gamma(s), \gamma(t))=|s-t| d(x, y)$ for $s, t \in[0,1]$. We call such a curve $\gamma$ minimal geodesic joining $x$ and $y$. Let $P_{t}(x, \cdot) \in \mathscr{P}(M), t \geq 0, x \in M$ be a semigroup of Markov kernels on $M$, where $\mathscr{P}(M)$ is the space of all Borel probability measures on $M$. We denote the action of the Markov kernel $P_{t}$ to $f: M \rightarrow \mathbb{R}$ bounded and measurable by $P_{t} f$. Similarly, the dual action of $P_{t}$ to $\mu \in \mathscr{P}(M)$ is denoted by $P_{t}^{*} \mu$. We denote the set of (bounded) Lipschitz functions by $C^{\text {Lip }}(M)$ and $C_{b}^{\text {Lip }}(M)$ respectively. Let us denote the local Lipschitz constant of $f \in C^{\operatorname{Lip}}(M)$ at $x$ by $|\nabla f|(x)$. That is,

$$
|\nabla f|(x)=\limsup _{y \rightarrow x} \frac{|f(y)-f(x)|}{d(x, y)} .
$$

Let $\operatorname{Lip}(f)$ stand for the (global) Lipschitz constant of $f$. Recall that we say a curve $(\gamma(r))_{r \in[0,1]}$ in a metric space $\left(Y, d_{Y}\right)$ is called absolutely continuous if there is a nonnegative integrable function $\varphi$ on $[0,1]$ such that $d_{Y}(\gamma(s), \gamma(t)) \leq \int_{s}^{t} \varphi(r) d r$ for any $0 \leq s \leq t \leq 1$. $\varphi$ can be chosen to be the metric derivative $|\dot{\gamma}|$ given by

$$
|\dot{\gamma}|(r):=\limsup _{s \rightarrow r} \frac{d_{Y}(\gamma(s), \gamma(r))}{|s-r|}
$$

(see [5, Theorem 1.1.2]). For $1 \leq p<\infty$, we denote the $L^{p}$-Wasserstein (pseudo-)distance on $\mathscr{P}(M)$ by $W_{p}$. That is,

$$
W_{p}(\mu, \nu):=\inf \left\{\|d\|_{L^{p}(\pi)} \mid \pi \text { is a coupling of } \mu \text { and } \nu\right\} .
$$

For each $\mu_{0}, \mu_{1} \in \mathscr{P}(M)$, there is a curve $(\mu(t))_{t \in[0,1]} \subset \mathscr{P}(M)$ such that $\mu(i)=\mu_{i}$ for $i=0,1$ and $W_{p}\left(\mu\left(r_{1}\right), \mu\left(r_{2}\right)\right)=\left|r_{1}-r_{2}\right| W_{p}\left(\mu_{0}, \mu_{1}\right)$ for any $r_{1}, r_{2} \in[0,1]$ (see [32, Corollary 1 and Proposition 1]). Let $\operatorname{Geo}(M)$ be the space of minimal geodesics parametrized by $[0,1]$ and $e_{t}: \operatorname{Geo}(M) \rightarrow M(t \in[0,1])$ the evaluation map given by $e_{t}(\gamma)=\gamma(t)$. By $[32$, Corollary 1 and Theorem 6], there exists a probability measure $\Gamma$ on $\operatorname{Geo}(M)$ such that $\left(e_{r}\right)_{\sharp} \Gamma=\mu(r)$ and

$$
\int_{\mathrm{Geo}(M)} d\left(\gamma\left(r_{1}\right), \gamma\left(r_{2}\right)\right)^{p} \Gamma(d \gamma)=\int_{\mathrm{Geo}(M)} \int_{r_{1}}^{r_{2}}|\dot{\gamma}|(u)^{p} d u \Gamma(d \gamma)=W_{p}\left(\mu\left(r_{1}\right), \mu\left(r_{2}\right)\right)^{p}
$$

for any $r_{1}, r_{2} \in[0,1]$ with $r_{1}<r_{2}$. we call such $\Gamma$ a dynamic optimal coupling of $\mu_{0}$ and $\mu_{1}$. 
We introduce some quantities we will use throughout this paper. Let $a:[0, \infty) \rightarrow$ $(0, \infty)$ and $b:(0, \infty) \rightarrow(0, \infty)$ be continuous functions. We define a measure $J$ on $[0, \infty)$ by $J(d x)=b(x)^{-1} d x$. We assume that $J$ is locally finite, that is, $J([0, \delta))<\infty$ for any $\delta>0$. Let $p, p_{*}, \beta, \beta_{*} \in(1, \infty)$ with $p^{-1}+p_{*}^{-1}=1, \beta^{-1}+\beta_{*}^{-1}=1$ and $\beta \leq p$. For $f: M \rightarrow \mathbb{R}$, we define the Hopf-Lax (or Hamilton-Jacobi) semigroup $\left(Q_{s} f\right)_{s \geq 0}$ by

$$
Q_{s} f(x):=\inf _{y \in X}\left[f(y)+\frac{s}{p}\left(\frac{d(x, y)}{s}\right)^{p}\right] .
$$

For the infinitesimal generator $\mathscr{L}$ of $P_{t}$, we suppose either of the following conditions: (A1) For any $f \in C_{b}^{\mathrm{Lip}}(M), t>0$ and $x \in M$, the following limit exists:

$$
\mathscr{L} P_{t} f(x):=\lim _{s \rightarrow 0} \frac{P_{t+s} f(x)-P_{t} f(x)}{s} .
$$

(A2) There is a locally finite reference measure $\mathfrak{m}$ on $M$ with $\operatorname{supp} \mathfrak{m}=M$ such that we can extend the action of $P_{t}$ to $L^{q}(\mathfrak{m})$ as a bounded operator for some $q \in[1, \infty)$ and the limit (2.4) exists in $L^{q}(\mathfrak{m})$ for any $f \in L^{q}(\mathfrak{m})$ and $t>0$.

The condition (A1) seems more restrictive, but the other assumptions can be rather weak and the proof of the main theorem is simpler under this condition. The condition (A2) requires some additional assumptions for the main theorem, but it naturally occurs when we are following a functional analytic approach. Such a situation arises in analysis on metric measure spaces where no (usual) differentiable structure is assumed. Under (A2), for two measurable functions $f$ and $g$ which belong to the same equivalence class in $L^{q}(\mathfrak{m})$, $\int_{M} f(y) d P_{t}(x, d y)=\int_{M} g(y) P_{t}(x, d y)$ holds $\mathfrak{m}$-a.e. $x \in M$. Thus, even under (A2), we always regard $P_{t} f$ for $f \in L^{q}(\mathfrak{m})$ as the integral by the Markov kernel of a representative of $f$.

We are interested in the following conditions:

(1) (Space-time $\left(L^{p}, L^{\beta}\right)$-Wasserstein control) For $\mu_{0}, \mu_{1} \in \mathscr{P}(M)$ and $0 \leq s<t$,

$$
W_{p}\left(P_{s}^{*} \mu_{0}, P_{t}^{*} \mu_{1}\right)^{\beta} \leq\left(\frac{1}{J([s, t])} \int_{[s, t]} \frac{J(d r)}{a(r)}\right)^{-\beta} W_{p}\left(\mu_{0}, \mu_{1}\right)^{\beta}+J([s, t])^{\beta} .
$$

(2) $\left(\left(L^{p_{*}}, L^{\beta_{*}}\right)\right.$-Bakry-Ledoux type gradient estimate) For $f \in C_{b}^{\operatorname{Lip}}(M), t>0$ and $x \in M$,

$$
\left|\nabla P_{t} f\right|(x)^{\beta_{*}} \leq a(t)^{\beta_{*}}\left(P_{t}\left(|\nabla f|^{p_{*}}\right)(x)^{\beta_{*} / p_{*}}-b(t)^{\beta_{*}}\left|\mathscr{L} P_{t} f(x)\right|^{\beta_{*}}\right)
$$

$(2)^{*}$ (2.6) holds for $t>0, x \in M$ and $f$ of the form $f=Q_{\delta} \tilde{f}$ with $\delta>0$ and $\tilde{f} \in C_{b}^{\operatorname{Lip}}(M)$.

(3) For any minimal geodesic $\gamma:[0,1] \rightarrow M, 0 \leq s \leq t$ and $f \in C_{b}^{\operatorname{Lip}}(M)$,

$$
\begin{aligned}
& \left|P_{t} f(\gamma(1))-P_{s} f(\gamma(0))\right| \\
\leq & \int_{0}^{1}\left(a(\xi(r))^{\beta} d(\gamma(0), \gamma(1))^{\beta}+\left(\frac{t-s}{b(\xi(r))}\right)^{\beta}\right)^{1 / \beta} P_{\xi(r)}\left(|\nabla f|^{p_{*}}\right)(\gamma(r))^{1 / p_{*}} d r
\end{aligned}
$$

where $\xi(r):=r t+(1-r) s$. 
Note that (2) implies (2)* since the function $f$ in $(2)^{*}$ belongs to $C_{b}^{\mathrm{Lip}}(M)$ (see Section 3.1). For (2) and (2)*, we consider a slightly modified version under (A2). When (2) or (2)* holds for $f \in C_{b}^{\mathrm{Lip}}(M) \cap L^{q}(\mathfrak{m})$ and $\mathfrak{m}$-a.e. $x \in M$ instead of $f \in C_{b}^{\mathrm{Lip}}(M)$ and $x \in M$, we denote those conditions by $(2)_{\text {ae }}$ or $(2)_{\text {ae }}^{*}$ respectively. Note that, when considering (2) ${ }^{*}, f=Q_{\delta} \tilde{f} \in C_{b}^{\mathrm{Lip}}(M)$ is automatic and $f \in L^{q}(m)$ holds if $\tilde{f} \in L^{q}(\mathfrak{m})$ and $\tilde{f} \geq 0$, or $\operatorname{supp} \tilde{f}$ is compact. We state our first main theorems of this paper as follows:

Theorem 2.1 Assume (A1). Then the conditions (1), (2)* and (3) are equivalent.

For considering the corresponding assertion under (A2), we introduce the following additional assumption associated with (A2):

(A3) With keeping $\mathfrak{m}$ and $q$ introduced in (A2), for any $\mu_{0}, \mu_{1} \in \mathscr{P}(M)$ with bounded supports and bounded densities with respect to $\mathfrak{m}$, there exists a $W_{p}$-minimal geodesic $\left(\mu_{r}\right)_{r \in[0,1]}$ such that $\mu_{t} \ll \mathfrak{m}$ and the density $\rho_{t}$ satisfies $\int_{A} \rho_{t}^{q_{*}} d \mathfrak{m}<\infty$ for each $t \in[0,1]$ and bounded $A \in \mathcal{B}(M)$, where $q_{*}$ is the Hölder conjugate of $q$.

Theorem 2.2 Assume (A2). Then the implication "(1) $\Rightarrow(3) \Rightarrow(2)_{\mathrm{ae}}^{*}$ " holds. In addition, the implication " $(2)_{\mathrm{ae}}^{*} \Rightarrow(\mathbf{1})$ " also holds true when (A3) holds.

For stating the full equivalence involving (2) instead of $(2)^{*}$, we introduce the following assumption on a regularization property of $P_{t}$.

(A4) $P_{t}$ is strong Feller, that is, $P_{t} f \in C_{b}(M)$ for any $t>0$ and any $f: M \rightarrow \mathbb{R}$ bounded and measurable.

Corollary 2.3 (i) Assume (A1) and (A4). Then (3) implies (2). In particular, (1), (2) and (3) are equivalent.

(ii) Assume (A2) and (A4). Then (3) implies (2) $)_{\mathrm{ae}}$. In particular, (1), (2) ae and (3) are equivalent if (A3) holds additionally.

As a special case of Corollary 2.3, we obtain the following:

Corollary 2.4 Let $K \in \mathbb{R}$ and $N \in(0, \infty)$.

(i) Assume (A1) and (A4). Then the following are equivalent:

(a) (1.4) holds for any $\mu_{0}, \mu_{1} \in \mathscr{P}(M)$ and $0<s<t$.

(b) (1.2) holds for any $f \in C_{b}^{\mathrm{Lip}}(M), t>0$ and $x \in M$.

(ii) Assume (A2), (A3) and (A4). Then the following are equivalent:

(a) (1.4) holds for any $\mu_{0}, \mu_{1} \in \mathscr{P}(M)$ and $0<s<t$

(b) (1.2) holds for any $f \in C_{b}^{\mathrm{Lip}}(M) \cap L^{q}(\mathfrak{m}), t>0$ and $\mathfrak{m}$-a.e. $x \in M$. 
Indeed, we obtain Corollary 2.4 from Theorem 2.1 and Theorem 2.2 with $p=\beta=2$, $a(t)=\mathrm{e}^{-K t}$ and $b(t)=\sqrt{\left(\mathrm{e}^{2 K t}-1\right) /(N K)}$.

The reader may think that it seems difficult to specify $a$ and $b$ in (2.5) when we have a bound of $W_{p}\left(P_{s}^{*} \mu_{0}, P_{t}^{*} \mu_{1}\right)$ involving $W_{p}\left(\mu_{0}, \mu_{1}\right), t$ and $s$. Even in such a case, we can find them by passing through our duality argument. See Remark 3.7.

We next state a equivalence between (1.2) and another Wasserstein control involving comparison functions. It is studied in [20] in connection with the reduced curvaturedimension condition introduced in [8]. Here we give a more direct proof under a slightly different assumptions. Let us introduce comparison functions as follows: For $\kappa \in \mathbb{R}$, we will define functions $\mathfrak{s}_{\kappa}, \mathfrak{c}_{\kappa}, \mathfrak{t}_{\kappa}$ on $\left[0,(\kappa \vee 0)^{-1 / 2} \pi\right] \cap[0, \infty)$ as follows:

$$
\mathfrak{s}_{\kappa}(u):=\frac{1}{\sqrt{\kappa}} \sin (\sqrt{\kappa} u), \quad \mathfrak{c}_{\kappa}(u):=\cos (\sqrt{\kappa} u), \quad \mathfrak{t}_{\kappa}(u):=\frac{\mathfrak{s}_{\kappa}(u)}{\mathfrak{c}_{\kappa}(u)} .
$$

When $\kappa>0$, there is no problem in this definition. When $\kappa=0$, we extend its definition naturally to the limit $\kappa \rightarrow 0$ of them, as usual. Even when $\kappa<0$, this definition makes sense by regarding trigonometric functions as complex functions. They take their values in $\mathbb{R}$ even in this case.

Theorem 2.5 Let $K \in \mathbb{R}$ and $N \in(0, \infty)$. When $K>0$, we suppose that $d(x, y)<$ $\pi \sqrt{(N-1) / K}$ holds for any $x, y \in M$.

(i) Assume (A1) and (A4). Then (1.2) holds for $f \in C_{b}^{\mathrm{Lip}}(M)$ and $t>0$ if and only if the following holds: For $0 \leq s<t$ and $\mu, \nu \in \mathscr{P}(M)$,

$$
\begin{aligned}
\mathfrak{s}_{K / N}^{2}\left(\frac{W_{2}\left(P_{s}^{*} \mu, P_{t}^{*} \nu\right)}{2}\right) \leq \mathrm{e}^{-K(s+t)} \mathfrak{s}_{K / N}^{2}\left(\frac{W_{2}(\mu, \nu)}{2}\right) & \\
& \quad+\frac{N}{2} \frac{1-\mathrm{e}^{-K(s+t)}}{K(s+t)}(\sqrt{t}-\sqrt{s})^{2} .
\end{aligned}
$$

(ii) Assume (A2), (A3) and (A4). Then (1.2) holds for $f \in C_{b}^{\mathrm{Lip}}(M) \cap L^{q}(\mathfrak{m}), t>0$ and $\mathfrak{m}$-a.e. $x \in M$ if and only if (2.8) holds for $0 \leq s<t$ and $\mu, \nu \in \mathscr{P}(M)$.

Note that the condition on the diameter in the last Theorem when $K>0$ holds in typical situations. See e.g. [14, 15] and [20, Remark 3.5 and Corollary 3.7] (cf. Remark 4.1).

Our final main theorem deals with the case when $M$ is a $m$-dimensional complete Riemannian manifold. We consider the diffusion process $\left((X(t))_{t \geq 0},\left(\mathbb{P}_{x}\right)_{x \in M}\right)$ generated by $\mathscr{L}=\Delta+Z$, where $Z$ is a smooth vector field. For $K \in \mathbb{R}$ and $N \in[m, \infty)$, we say that the Bakry-Émery Ricci tensor associated with $\mathscr{L}$ satisfies the $(K, N)$-curvature-dimension bound if the following holds:

$$
\operatorname{Ric}-(\nabla Z)^{b}-\frac{1}{N-m} Z \otimes Z \geq K
$$

where $(\nabla Z)^{b}$ is a symmetrization of $\nabla Z$ as $(0,2)$-tensor. When $N=m$, we interpret $(2.9)$ as $Z=0$ and Ric $\geq K$. Let $P_{t}(x, \cdot)$ be given by distributions of the diffusion process $X(t): P_{t}(x, \cdot)=\mathbb{P}_{x} \circ X(t)^{-1}$. In this case, we will obtain the following: 
Theorem 2.6 Assume $p \geq 2$ and (2.9) for some $K \in \mathbb{R}$ and $N \in[m, \infty)$.

(i) For $t>s>0$ and $\mu_{0}, \mu_{1} \in \mathscr{P}(M)$,

$$
\begin{aligned}
W_{p}\left(P_{s} \mu_{0}, P_{t} \mu_{1}\right)^{2} \leq\left(\frac{1}{J_{N+p-2}([s, t])} \int_{s}^{t} \mathrm{e}^{K r} J_{N+p-2}(d r)\right)^{-2} & W_{p}\left(\mu_{0}, \mu_{1}\right)^{2} \\
& +J_{N+p-2}([s, t])^{2},
\end{aligned}
$$

where $J$. is as defined in (1.5).

(ii) For $t>0$ and $f \in C_{b}^{\mathrm{Lip}}(M)$,

$$
\left|\nabla P_{t} f\right|(x)^{2} \leq \mathrm{e}^{-2 K t} P_{t}\left(|\nabla f|^{p_{*}}\right)^{2 / p_{*}}-\frac{1-\mathrm{e}^{-2 K t}}{(N+p-2) K}\left|\mathscr{L} P_{t} f\right|^{2} .
$$

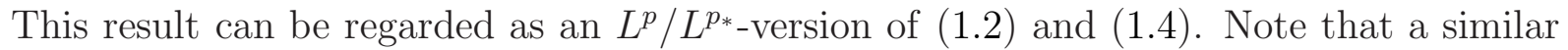
argument implies an estimate of transportation cost involving a comparison function (see Theorem 4.11).

The rest of this section consists of a series of remarks concerning with Theorem 2.1 and Theorem 2.2, including a discussion on sufficient conditions of assumptions in these theorems (Remark 2.8 and Remark 2.9).

Remark 2.7 For both (2.5) and (2.6), the inequality becomes stronger as $p$ increases (or $p_{*}$ decreases). For (2.5), This is based on the fact that (2.5) holds for any $\mu_{0}, \mu_{1} \in \mathcal{P}(M)$ if it does for any Dirac measures (see Lemma 3.4 below). Then the problem is reduced to an easy application of the Hölder inequality. As a by-product of this observation, When both $\mu_{0}$ and $\mu_{1}$ are Dirac measure, (2.5) yields the same estimate even when $1 \leq p<\beta$. For (2.6), this is an easy consequence of the Hölder inequality. Here we do not require the fact $p_{*} \leq \beta_{*}$. Note that these arguments do not require the conclusion of Theorem 2.1 or Theorem 2.2. Note also that, in the $L^{p} / L^{p_{*}}$-estimates in Theorem 2.6, the constant corresponding to $b(t)$ does depend on $p$. Thus it is not clear whether the same implication still holds or not.

Remark 2.8 The strong Feller property we assumed in Corollary 2.3 holds for the heat semigroup associated with the (quadratic) Cheeger energy functional on a metric measure space with a Riemannian lower Ricci curvature bound (see [4, Theorem 6.1 (iii)] and [1, Theorem 7.1 (iii)]). More generally, when the semigroup is associated with a Dirichlet form, it is known that (1.1) or (1.3) yields the strong Feller property under some regularity assumptions [2, Theorem 3.17]. Since either (1.1) or (1.3) immediately follows from (1.2) or (1.4), the strong Feller property is closely related with our conditions.

Remark 2.9 The assumption (A3) is satisfied for any $q \in[1, \infty)$ if $(M, d, \mathfrak{m})$ enjoys the curvature-dimension condition $C D(K, \infty)$ in the sense of [38] for some $K \in \mathbb{R}$ (see [36]). Note that, even in this framework, our semigroup $P_{t}$ is not necessarily the one studied in [1, 3, 4], which is associated with the (quadratic) Cheeger energy. 
Remark 2.10 In [29, 40], the monotonicity of normalized $\mathcal{L}$-transportation cost between two heat distributions on a backward Ricci flow is studied. It can be written in the following form: For $s_{1}<s_{2}$ and $t_{1}, t_{2}>0$ with $t_{2} / t_{1}=s_{2} / s_{1}$,

$$
\mathcal{T}_{L}\left(\mu_{s_{1}+t_{1}}, s_{1}+t_{1} ; \mu_{s_{2}+t_{2}}, s_{2}+t_{2}\right) \leq \mathcal{T}_{L}\left(\mu_{s_{1}}, s_{1} ; \mu_{s_{2}}, s_{2}\right)+2 m\left(\sqrt{t_{2}}-\sqrt{t_{1}}\right)^{2},
$$

where $m$ is the dimension of the manifold, $\mu_{t}$ is the heat distribution at time $t$,

$$
\mathcal{T}_{L}(\mu, s ; \nu, t):=\inf \left\{2(\sqrt{t}-\sqrt{s}) \int L(x, s ; y, t) \pi(d x d y) \mid \pi: \text { coupling of } \mu \text { and } \nu\right\}
$$

and $L$ is Perelman's $\mathcal{L}$-distance. It looks very similar to (1.4) with $K=0$.

Remark 2.11 When $s=0, \mu_{0}=\delta_{y}$ and $\mu_{1}=\delta_{x}$, the inequality (2.8) becomes the following form:

$$
\begin{aligned}
\mathfrak{s}_{K / N}^{2}\left(\frac{W_{2}\left(\delta_{y}, P_{t} \delta_{x}\right)}{2}\right) & =\mathfrak{s}_{K / N}^{2}\left(\frac{1}{2}\left(\int_{M} d(y, z)^{2} P_{t}(x, d z)\right)^{1 / 2}\right) \\
& \leq \mathrm{e}^{-K t} \mathfrak{s}_{K / N}^{2}\left(\frac{d(y, x)}{2}\right)+\frac{N t}{2}+o(t) .
\end{aligned}
$$

By applying the Hölder inequality to bound $W_{1}$ by $W_{2}$, the last inequality formally implies the following estimate by taking a derivative at $t=0$ :

$$
(\mathscr{L} d(y, \cdot))(x) \leq \frac{N}{\mathfrak{t}_{K / N}(d(x, y))}
$$

It corresponds to the Laplacian comparison theorem on complete Riemannian manifolds, but slightly weaker (it was sharp if we could replace $N$ with $N-1$ ). By the same argument based on (1.4) instead of (2.8), the sharp estimate follows when $K=0$. By using an estimate in Theorem 4.11 below (with $p=2$ ), we can recover the sharp estimate for $K \in \mathbb{R}$, but it is shown only on a complete Riemannian manifold (see e.g. [27, Lemma 2.1] for a more direct proof of the Laplacian comparison theorem for $\mathscr{L}=\Delta+Z$ ).

\section{Proof of DUALities}

Before going into the proof, we review known properties of the Hopf-Lax semigroup.

\subsection{Reminder of the Hopf-LAX SEmigroup}

Recall that the Hopf-Lax semigroup is defined as in (2.3). It is immediate from the definition that $Q_{s} f$ is non-increasing in $s$ and

$$
\inf _{y \in M} f(y) \leq Q_{s} f(x) \leq f(x) .
$$

When $f$ is bounded, we can easily observe

$$
Q_{s} f(x)=\inf \left\{\begin{array}{l|l}
f(y)+\frac{s}{p}\left(\frac{d(x, y)}{s}\right)^{p} & \begin{array}{l}
y \in M \\
d(x, y) \leq s\left(\frac{p(\sup f-\inf f)}{s}\right)^{1 / p}
\end{array}
\end{array}\right\}
$$


(see e.g. [21, Proposition A.3 (1)]). Therefore, if $f$ is bounded with bounded support, then $Q_{s} f$ shares the same property. In addition, by virtue of $(3.2), Q_{s} f \in C_{b}^{\mathrm{Lip}}(M)$ holds if $f$ is bounded. Again by (3.2), we have

$$
\lim _{t \rightarrow 0} Q_{t} f(x) \geq \liminf _{y \rightarrow x} f(y),
$$

where the limit in the left hand side exists since $Q_{t} f(x)$ is monotone in $t$. This estimate together with (3.1) yields that $\lim _{s \downarrow 0} Q_{s} f(x)=f(x)$ holds for each fixed $x \in M$ if $f$ is lower semi-continuous. When $f \in C_{b}^{\mathrm{Lip}}(M)$, the same argument as in the proof of $[16$, Theorem 2.1 (iv)] yields

$$
\begin{aligned}
\left|Q_{s} f(x)-Q_{s} f(y)\right| & \leq \operatorname{Lip}(f) d(x, y), \\
\left|Q_{s^{\prime}} f(x)-Q_{s} f(x)\right| & \leq \frac{\operatorname{Lip}(f)^{p_{*}}}{p_{*}}\left|s^{\prime}-s\right|
\end{aligned}
$$

for each $x, y \in M, s, s^{\prime}>0$. As an important property of $Q_{s} f$, it is a solution to a Hamilton-Jacobi equation in the following sense:

$$
\frac{\partial^{+}}{\partial s} Q_{s} f(x)=-\frac{1}{p_{*}}\left|\nabla Q_{s} f\right|^{p_{*}}(x)
$$

for any $x \in M$ and $s>0$ (see [6, 21], [28, Theorem 3.6 and Theorem 3.8] and references therein). Note that we use the property that $M$ is a geodesic space to obtain the equality (3.6) while an inequality " $\leq$ " holds without this assumption. By [28, Lemma 3.3 and Proposition 3.4] (see [3, 6] also) and (3.6), the function $x \mapsto\left|\nabla Q_{s} f\right|(x)$ is upper semicontinuous. This fact works as a sort of regularization of $|\nabla f|$.

\subsection{From Wasserstein CONTROL TO GRAdient estimates}

In this subsection, we will give the proof of the implication " $(1) \Rightarrow(3) \Rightarrow(2)^{*}$ " in Theorem 2.1 and the corresponding assertions in Theorem 2.2, Corollary 2.3 and Theorem 2.5. The argument is separated into Proposition 3.1, Proposition 3.2 and Lemma 3.3. We will show all these implications at the end of this section.

Proposition 3.1 Let $A:[0, \infty)^{2} \rightarrow(0, \infty)$ and $B:[0, \infty)^{2} \rightarrow(0, \infty)$ continuous functions satisfying $A(s, t)=A(t, s)$ and $B(s, t)=B(t, s)$. Assume that $B(s, t)=0$ if and only if $s=t$. In addition, we assume $A(t, t)=a(t)$ and

$$
b(t)=\lim _{s \rightarrow t} \frac{|s-t|}{B(s, t)}
$$

for $t \in[0, \infty)$, where $a, b$ is as introduced in Section 2. Suppose the following inequality holds:

$$
W_{p}\left(P_{s}^{*} \delta_{x}, P_{t}^{*} \delta_{y}\right)^{\beta} \leq A(s, t)^{\beta} d(x, y)^{\beta}+B(s, t)^{\beta}
$$


for any $x, y \in M$ and $0 \leq s<t$. Then, for any absolutely continuous curve $(\xi(r), \gamma(r))_{r \in[0,1]}$ in $(0, \infty) \times M$ and $f \in C_{b}^{\mathrm{Lip}}(M)$,

$$
\begin{aligned}
\mid P_{\xi(1)} f(\gamma(1))- & P_{\xi(0)} f(\gamma(0)) \mid \\
& \leq \int_{0}^{1}\left((a(\xi(r))|\dot{\gamma}|(r))^{\beta}+\left(\frac{\left|\xi^{\prime}(r)\right|}{b(\xi(r))}\right)^{\beta}\right)^{1 / \beta} P_{\xi(r)}\left(|\nabla f|^{p_{*}}\right)(\gamma(r))^{1 / p_{*}} d r .
\end{aligned}
$$

In particular, the condition (3) holds.

Note that neither (A1) nor (A2) is required in Proposition 3.1.

Proof. We first claim that $(t, x) \mapsto P_{t} f(x)$ is locally Lipschitz for any $f \in C_{b}^{\mathrm{Lip}}(M)$. Indeed, (3.7) yields that $P_{t}^{*} \delta_{z}$ is locally Lipschitz in $(t, z)$ with respect to $W_{p}$ by the assumption on $A$ and $B$. By the Hölder inequality, the same holds for $W_{1}$. Then the claim follows from the Kantorovich-Rubinstein duality (see [41, Remark 6.5] for instance). Note that the claim implies that $(t, z) \mapsto P_{t}^{*} \delta_{z}$ is continuous on $(0, \infty) \times M$ with respect to the topology of weak convergence. Let $(\xi(s), \gamma(s))_{s \in[0,1]}$ be an absolutely continuous curve in $(0, \infty) \times M$. Then our claim implies that $P_{\xi(s)} f(\gamma(s))$ is absolutely continuous in $s$. Thus it is differentiable a.e. with respect to the Lebesgue measure on $[0,1]$.

Let $s \in[0,1)$ where $s \mapsto P_{\xi(s)} f(\gamma(s))$ is differentiable and take $\varepsilon>0$ such that $s+\varepsilon \in[0,1]$. Let $\pi_{s}^{\varepsilon} \in \mathscr{P}(M \times M)$ be a minimizer of $W_{p}\left(P_{\xi(s)} \delta_{\gamma(s)}, P_{\xi(s+\varepsilon)} \delta_{\gamma(s+\varepsilon)}\right)$. Then we have

$$
\left|P_{\xi(s+\varepsilon)} f(\gamma(s+\varepsilon))-P_{\xi(s)} f(\gamma(s))\right| \leq \int_{M \times M}|f(z)-f(w)| \pi_{s}^{\varepsilon}(d z d w) .
$$

Take $r>0$, which is specified later, and set

$$
G_{r} f(z):=\sup _{z^{\prime} ; d\left(z, z^{\prime}\right) \in(0, r)} \frac{\left|f(z)-f\left(z^{\prime}\right)\right|}{d\left(z, z^{\prime}\right)} .
$$

Then we have

$$
\begin{aligned}
\int_{M \times M}|f(z)-f(w)| \pi_{s}^{\varepsilon}(d z d w)= & \int_{M \times M}|f(z)-f(w)| 1_{\{d(z, w) \leq r\}} \pi_{s}^{\varepsilon}(d z d w) \\
& +\int_{M \times M}|f(z)-f(w)| 1_{\{d(z, w)>r\}} \pi_{s}^{\varepsilon}(d z d w) \\
\leq & \int_{M \times M} G_{r} f(z) d(z, w) \pi_{s}^{\varepsilon}(d z d w)+2\|f\|_{\infty} \pi_{s}^{\varepsilon}(d>r) \\
\leq & P_{\xi(s)}\left(\left(G_{r} f\right)^{p_{*}}\right)(\gamma(s))^{1 / p_{*}} W_{p}\left(P_{\xi(s)} \delta_{\gamma(s)}, P_{\xi(s+\varepsilon)} \delta_{\gamma(s+\varepsilon)}\right) \\
& \quad+\frac{2\|f\|_{\infty}}{r^{p}} W_{p}\left(P_{\xi(s)} \delta_{\gamma(s)}, P_{\xi(s+\varepsilon)} \delta_{\gamma(s+\varepsilon)}\right)^{p},
\end{aligned}
$$

where $\|f\|_{\infty}=\sup _{x \in M}|f(x)|$. Let us choose $r=r(s, \varepsilon)$ by

$$
r(s, \varepsilon):=W_{p}\left(P_{\xi(s)} \delta_{\gamma(s)}, P_{\xi(s+\varepsilon)} \delta_{\gamma(s+\varepsilon)}\right)^{1 /\left(2 p_{*}\right)} .
$$


Applying (3.7) to (3.9) and substituting it into (3.8), we obtain

$$
\begin{aligned}
& \quad\left|P_{\xi(s+\varepsilon)} f(\gamma(s+\varepsilon))-P_{\xi(s)} f(\gamma(s))\right| \\
& \leq P_{\xi(s)}\left(\left(G_{r} f\right)^{p_{*}}\right)(\gamma(s))^{1 / p_{*}}\left(A(\xi(s), \xi(s+\varepsilon))^{\beta} d(\gamma(s), \gamma(s+\varepsilon))^{\beta}+B(\xi(s), \xi(s+\varepsilon))^{\beta}\right)^{1 / \beta} \\
& \quad+2\|f\|_{\infty} W_{p}\left(P_{\xi(s)} \delta_{\gamma(s)}, P_{\xi(s+\varepsilon)} \delta_{\gamma(s+\varepsilon)}\right)^{(p+1) / 2} .
\end{aligned}
$$

Note that $W_{p}\left(P_{\xi(s)} \delta_{\gamma(s)}, P_{\xi(s+\varepsilon)} \delta_{\gamma(s+\varepsilon)}\right)=O(\varepsilon)$ as $\varepsilon \downarrow 0$ by (3.7). In particular, $r \rightarrow 0$ as $\varepsilon \downarrow 0$. We divide (3.10) by $\varepsilon$ and let $\varepsilon \downarrow 0$. Since $G_{r} f \leq \operatorname{Lip}(f)<\infty$, the dominated convergence theorem yields

$$
\left|\frac{\partial}{\partial s} P_{\xi(s)} f(\gamma(s))\right| \leq P_{\xi(s)}\left(|\nabla f|^{p_{*}}\right)(\gamma(s))^{1 / p_{*}}\left((a(\xi(s))|\dot{\gamma}|(s))^{\beta}+\left(\frac{\left|\xi^{\prime}(s)\right|}{b(\xi(s))}\right)^{\beta}\right)^{1 / \beta} .
$$

Thus the assertion holds by integrating the last inequality with respect to $s$ on $[0,1]$.

Proposition 3.2 Assume (3). Then $(t, x) \mapsto P_{t} f(x)$ is Lipschitz on $[a, b] \times M$ for any $0<a<b$. In addition,

$$
\left|\nabla P_{t} f\right|(x)^{\beta_{*}} \leq a(t)^{\beta_{*}}\left(P_{t}\left(|\nabla f|^{p_{*}}\right)(x)^{\beta_{*} / p_{*}}-b(t)^{\beta_{*}}\left|\frac{\partial}{\partial t} P_{t} f(x)\right|^{\beta_{*}}\right)
$$

holds for $f$ of the form $f=Q_{\delta} \tilde{f}$ with $\delta>0$ and $\tilde{f} \in C_{b}^{\mathrm{Lip}}(M), x \in M$ and $t>0$ at which $s \mapsto P_{s} f(x)$ is differentiable. Moreover, the same conclusion holds for any $f \in C_{b}^{\mathrm{Lip}}(M)$ under (A4).

Proof. Let $f \in C_{b}^{\mathrm{Lip}}(M), t, s>0$ with $t \neq s$ and $x, y \in M$ with $x \neq y$. Take a minimal geodesic $\gamma$ in $M$ from $x$ to $y$ and let $\xi(r):=(t-s) r+s$. Then (2.7) yields

$$
\begin{aligned}
& \left|P_{t} f(y)-P_{s} f(x)\right| \\
& \quad \leq \int_{0}^{1}\left((a(\xi(r)) d(x, y))^{\beta}+\left(\frac{|t-s|}{b(\xi(r))}\right)^{\beta}\right)^{1 / \beta} P_{\xi(r)}\left(|\nabla f|^{p_{*}}\right)(\gamma(r))^{1 / p_{*}} d r .
\end{aligned}
$$

Because $|\nabla f| \leq \operatorname{Lip}(f),(3.11)$ implies the claimed Lipschitz continuity. Note that $(t, x) \mapsto$ $P_{t}^{*} \delta_{x}$ is also continuous on $(0, \infty) \times M$ with respect to the topology of weak convergence by the Kantorovich-Rubinstein duality. Let $\alpha \in \mathbb{R} \backslash\{0\}$, define $\sigma_{*} \in\{ \pm 1\}$ by

$$
\sigma_{*}:= \begin{cases}1 & \text { if } \limsup _{y \rightarrow x} \frac{P_{t} f(y)-P_{t} f(x)}{d(y, x)}=\left|\nabla P_{t} f\right|(x), \\ -1 & \text { otherwise }\end{cases}
$$

and set $\alpha_{*}:=\sigma_{*} \alpha$. Take $y \in M$ satisfying $0<|\alpha| d(x, y)<t$ and set $s:=t-\alpha_{*} d(x, y)>0$. Then (3.11) yields

$$
\begin{aligned}
& \left|\frac{P_{t} f(y)-P_{s} f(x)}{d(x, y)}\right| \\
& \quad \leq \int_{0}^{1}\left(1+\left(\frac{|\alpha|}{a(\xi(r)) b(\xi(r))}\right)^{\beta}\right)^{1 / \beta} a(\xi(r)) P_{\xi(r)}\left(|\nabla f|^{p_{*}}\right)(\gamma(r))^{1 / p_{*}} d r .
\end{aligned}
$$


Now we claim that $P_{\xi(r)}\left(|\nabla f|^{p_{*}}\right)(\gamma(r))^{1 / p_{*}}$ is upper semi-continuous in $r$ if either $f=Q_{\delta} \tilde{f}$ for some $\delta>0$ and $\tilde{f} \in C_{b}^{\text {Lip }}(M)$, or (A4) holds. In the former case, $|\nabla f|$ is upper semi-continuous as reviewed in Section 3.1. Since $Q_{\delta^{\prime}}\left(-|\nabla f|^{p_{*}}\right) \in C_{b}(M)$ holds for $\delta^{\prime}>0,(3.1)$ and (3.3) yield

$$
\begin{aligned}
\limsup _{r^{\prime} \rightarrow r} P_{\xi\left(r^{\prime}\right)}\left(|\nabla f|^{p_{*}}\right)\left(\gamma\left(r^{\prime}\right)\right)^{1 / p_{*}} & \leq \limsup _{\delta^{\prime} \downarrow 0}\left(\lim _{r^{\prime} \rightarrow r} P_{\xi\left(r^{\prime}\right)}\left(-Q_{\delta^{\prime}}\left(-|\nabla f|^{p_{*}}\right)\right)\left(\gamma\left(r^{\prime}\right)\right)^{1 / p_{*}}\right) \\
& \leq P_{\xi(r)}\left(|\nabla f|^{p_{*}}\right)(\gamma(r))^{1 / p_{*}} .
\end{aligned}
$$

In the latter case, $P_{\varepsilon} f \in C_{b}(M)$ for arbitrarily small $\varepsilon>0$. Since $(t, x) \mapsto P_{t}^{*} \delta_{x}$ is continuous, $P_{\xi(r)}\left(|\nabla f|^{p_{*}}\right)(\gamma(r))$ is continuous in $r$.

To conclude (2.6) from (3.12), we consider the left hand side of (3.12). By our choice of $s, t$ and $\sigma_{*}$, we have

$$
\begin{aligned}
\limsup _{y \rightarrow x} \sigma_{*} \frac{P_{t} f(y)-P_{s} f(x)}{d(x, y)} & =\limsup _{y \rightarrow x}\left(\sigma_{*} \frac{P_{t} f(y)-P_{t} f(x)}{d(x, y)}+\alpha \frac{P_{t} f(x)-P_{s} f(x)}{t-s}\right) \\
& =\left|\nabla P_{t} f\right|(x)+\alpha \frac{\partial}{\partial t} P_{t} f(x) .
\end{aligned}
$$

Thus we obtain

$$
\begin{aligned}
\alpha \frac{\partial}{\partial t} P_{t} f(x)+\left|\nabla P_{t} f\right|(x) & \leq \limsup _{y \rightarrow x}\left|\frac{P_{t} f(y)-P_{s} f(x)}{d(x, y)}\right| \\
& \leq\left(1+\left(\frac{|\alpha|}{a(t) b(t)}\right)^{\beta}\right)^{1 / \beta} a(t) P_{t}\left(|\nabla f|^{p_{*}}\right)(x)^{1 / p_{*}}
\end{aligned}
$$

Then the conclusion follows by optimizing over $\alpha$.

To deal with the case under (A2), we prepare the following lemma.

Lemma 3.3 Assume (A2) and that $(t, x) \mapsto P_{t} f(x)$ is Lipschitz on $[a, b] \times M$ for any $0<a<b$. Then, for each $t>0, P_{t} f(x)$ is differentiable at $t \mathfrak{m}$-a.e. $x \in M$.

The proof of this lemma goes in a similar way as the one for the corresponding assertion in the proof of [20, Theorem 4.4].

Proof. For each $x \in M, t \mapsto P_{t} f(x)$ is differentiable for a.e. $t$ with respect to the Lebesgue measure. The Fubini theorem yields that the set $\tilde{I} \subset(0, \infty)$ defined by

$$
\tilde{I}:=\left\{t \in(0, \infty) \mid P_{t} f(x) \text { is differentiable at } t \text { for } \mathfrak{m} \text {-a.e. } x \in M\right\}^{c}
$$

is of null Lebesgue measure. The proof will be completed once we prove $\tilde{I}=\emptyset$. Let $t \in(0, \infty)$. Then we have $s \in \tilde{I}^{c}$ with $s<t$. Note that (A2) implicitly yields that $P_{t}(x, A)=0$ holds for $\mathfrak{m}$-a.e. $x \in M$ for any measurable $A \subset M$ with $\mathfrak{m}(A)=0$. Since $(t, x) \mapsto P_{t} f(x)$ is Lipschitz, the dominated convergence theorem implies

$$
P_{t-s}\left(\frac{\partial}{\partial t} P_{s} f\right)=P_{t-s}\left(\lim _{h \rightarrow 0} \frac{P_{s+h} f-P_{s} f}{h}\right)=\frac{\partial}{\partial t} P_{t} f
$$

$\mathfrak{m}$-a.e. and hence $P_{t} f(x)$ is differentiable at $t$ for $\mathfrak{m}$-a.e. $x \in M$. It means $t \notin \tilde{I}$ and hence the assertion holds. 
Now we argue the implications " $(1) \Rightarrow(3) \Rightarrow(2)^{*}$ " in Theorem 2.1, the corresponding implication in Theorem 2.2 and Corollary 2.3. In all these cases, the implication " $(1) \Rightarrow$ (3)" follows immediately from Proposition 3.1 by taking $A$ and $B$ as follows: for $s<t$,

$$
A(s, t):=\left(\frac{1}{J([s, t])} \int_{[s, t]} \frac{J(d r)}{a(r)}\right)^{-1}, \quad B(s, t):=J([s, t]) .
$$

By (A1), the implications " $(3) \Rightarrow(2)^{*}$ " in Theorem 2.1 and "(3) $\Rightarrow(2)$ " in Corollary $2.3(\mathrm{i})$ is a direct consequence of Proposition 3.2. Under (A2), for $f \in C_{b}^{\mathrm{Lip}}(M) \cap$ $L^{q}(\mathfrak{m}), \frac{\partial}{\partial t} P_{t} f(x)=\mathscr{L} P_{t} f(x)$ holds $\mathfrak{m}$-a.e. $x \in M$ if the derivation in the left hand side is defined in the classical sense $\mathfrak{m}$-a.e. Thus Proposition 3.2 and Lemma 3.3 yields the implications " $(3) \Rightarrow(2)_{\mathrm{ae}}^{*}$ " in Theorem 2.2 and " $(3) \Rightarrow(2)_{\mathrm{ae}}$ " in Corollary 2.3 (ii).

Finally, we briefly discuss two implications "(2.8) $\Rightarrow(2.6)$ " in Theorem 2.5 (i) and (ii). This can be reduced to similar arguments because $\mathfrak{s}_{K / N}(u) \sim u$ as $u \rightarrow 0$.

\subsection{FROM GRADIENT ESTIMATE TO WASSERSTEIN CONTROLS}

For the rest of the proof of Theorem 2.1, Theorem 2.2 and Theorem 2.5, the estimate of the Wasserstein distance between Markov kernels given in Proposition 3.5 and Proposition 3.6 below is essential. We begin with the following auxiliary lemma.

Lemma 3.4 Let $0<t^{\prime} \leq t^{\prime \prime}$ and $C_{1}, C_{2} \geq 0$. If

$$
W_{p}\left(P_{t^{\prime}}^{*} \mu_{0}, P_{t^{\prime \prime}}^{*} \mu_{1}\right)^{\beta} \leq C_{1} W_{p}\left(\mu_{0}, \mu_{1}\right)^{\beta}+C_{2}
$$

holds whenever $\mu_{0}, \mu_{1}$ are Dirac measures, then the same holds for any $\mu_{0}, \mu_{1} \in \mathscr{P}(M)$.

Proof. The proof goes along the same line as [25, Lemma 3.3] (cf. [41, Theorem 4.8]). Thus we omit some technical details. For each $x_{1}, x_{2} \in M$, take an optimal coupling $\tilde{\pi}_{x_{1} x_{2}} \in \mathscr{P}(M)$ of $P_{t^{\prime}}^{*} \delta_{x_{1}}$ and $P_{t^{\prime \prime}}^{*} \delta_{x_{2}}$. Let $\pi \in \mathscr{P}\left(M^{2}\right)$ be an optimal coupling of $\mu_{0}$ and $\mu_{1}$ and define $\tilde{\pi} \in \mathscr{P}\left(M^{2}\right)$ by

$$
\tilde{\pi}(A):=\int_{M^{2}} \tilde{\pi}_{x_{1} x_{2}}(A) \pi\left(d x_{1} d x_{2}\right)
$$

Then the assumption and the Minkowski inequality for $L^{p / \beta}$-norm yield

$$
\begin{aligned}
W_{p}\left(P_{t^{\prime}}^{*} \mu_{0}, P_{t^{\prime \prime}}^{*} \mu_{1}\right)^{\beta} & \leq\left(\int_{M^{2}} W_{p}\left(P_{t^{\prime}}^{*} \delta_{x_{1}}, P_{t^{\prime \prime}}^{*} \delta_{x_{2}}\right)^{p} \pi\left(d x_{1} d x_{2}\right)\right)^{\beta / p} \\
& \leq\left(\int_{M^{2}}\left(C_{1} d\left(x_{1}, x_{2}\right)^{\beta}+C_{2}\right)^{p / \beta} \pi\left(d x_{1} d x_{2}\right)\right)^{\beta / p} \\
& \leq C_{1}\left\|d^{\beta}\right\|_{L^{p / \beta}(\pi)}+C_{2} \\
& =C_{1} W_{p}\left(\mu_{0}, \mu_{1}\right)^{\beta}+C_{2} .
\end{aligned}
$$

Hence the conclusion holds. 
Proposition 3.5 Let $0 \leq s \leq t$. Let $\eta:[0,1] \rightarrow[0,1]$ and $\xi:[0,1] \rightarrow[s, t]$ be $C^{1}$ increasing surjections. Assume (A1) and (2)*. Then, for $\mu_{0}, \mu_{1} \in \mathscr{P}(M)$,

$$
W_{p}\left(P_{s}^{*} \mu_{0}, P_{t}^{*} \mu_{1}\right)^{\beta} \leq \int_{0}^{1}\left(a(\xi(r))^{\beta} W_{p}\left(\mu_{0}, \mu_{1}\right)^{\beta} \eta^{\prime}(r)^{\beta}+\left(\frac{\xi^{\prime}(r)}{b(\xi(r))}\right)^{\beta}\right) d r .
$$

Proof. By virtue of Lemma 3.4, it suffices to show the assertion when $\mu_{0}=\delta_{x_{0}}, \mu_{1}=\delta_{x_{1}}$, $x_{0}, x_{1} \in M$. However, for later use, we argue with general $\mu_{0}, \mu_{1} \in \mathscr{P}(M)$ for a while.

Take a $W_{p}$-minimal geodesic $(\mu(r))_{r \in[0,1]}$ from $\mu_{0}$ to $\mu_{1}$. Let $t^{\prime}, t^{\prime \prime} \in[0,1]$ with $t^{\prime \prime}>t^{\prime}$ and set $h=t^{\prime \prime}-t^{\prime}$. Then the Kantorovich duality yields

$$
\begin{aligned}
\frac{1}{p} & \left(\frac{W_{p}\left(P_{\xi\left(t^{\prime}\right)}^{*} \mu\left(\eta\left(t^{\prime}\right)\right), P_{\xi\left(t^{\prime \prime}\right)}^{*} \mu\left(\eta\left(t^{\prime \prime}\right)\right)\right)}{h}\right)^{p} \\
& =\frac{1}{h} \sup _{f \in C_{b}^{\mathrm{Lip}}(M)}\left[\int_{M} Q_{h} f d P_{\xi\left(t^{\prime \prime}\right)}^{*} \mu\left(\eta\left(t^{\prime \prime}\right)\right)-\int_{M} f d P_{\xi\left(t^{\prime}\right)}^{*} \mu\left(\eta\left(t^{\prime}\right)\right)\right] \\
& =\frac{1}{h} \sup _{f \in C_{b}^{\mathrm{Lip}}(M)}\left[\int_{M} P_{\xi\left(t^{\prime \prime}\right)}\left(Q_{h} f\right) d \mu\left(\eta\left(t^{\prime \prime}\right)\right)-\int_{M} P_{\xi\left(t^{\prime}\right)} f d \mu\left(\eta\left(t^{\prime}\right)\right)\right] .
\end{aligned}
$$

Let $\Gamma$ be a dynamic optimal coupling associated with $(\mu(r))_{r \in[0,1]}$. Note that (2.6) is available for $Q_{r} f$ instead of $f$ for $r>0$ since we assume (2)*. With keeping this fact in mind, for $r_{i} \in[0, h]$ and $s_{i}, t_{i} \in\left[t^{\prime}, t^{\prime \prime}\right](i=1,2)$, we have

$$
\begin{aligned}
& \left|\int_{M} P_{\xi\left(t_{2}\right)} Q_{r_{2}} f d \mu\left(\eta\left(s_{2}\right)\right)-\int_{M} P_{\xi\left(t_{1}\right)} Q_{r_{1}} f d \mu\left(\eta\left(s_{1}\right)\right)\right| \\
& \leq\left|\int_{\text {Geo }(M)} \int_{\eta\left(s_{1}\right)}^{\eta\left(s_{2}\right)}\right| \nabla P_{\xi\left(t_{2}\right)} Q_{r_{2}} f|(\gamma(s))| \dot{\gamma}|(s) d s \Gamma(d \gamma)| \\
& \quad+\left|\int_{M}\left(P_{\xi\left(t_{2}\right)} Q_{r_{2}} f-P_{\xi\left(t_{1}\right)} Q_{r_{1}} f\right) d \mu\left(\eta\left(s_{1}\right)\right)\right| \\
& \quad+\left|\int_{M} \int_{\xi\left(t_{1}\right)}^{\xi\left(t_{2}\right)} \mathscr{L} P_{t} Q_{r_{1}} f d t d \mu\left(\eta\left(s_{1}\right)\right)\right| \\
& \leq \operatorname{Lip}(f) a\left(\xi\left(t_{2}\right)\right) W_{p}\left(\mu\left(\eta\left(s_{2}\right)\right), \mu\left(\eta\left(s_{1}\right)\right)\right)+\frac{\operatorname{Lip}(f)^{p_{*}}}{p_{*}}\left|r_{2}-r_{1}\right| \\
& \leq \operatorname{Lip}(f) a\left(\xi\left(t_{2}\right)\right) W_{p}\left(\mu_{0}, \mu_{1}\right)\left|\eta\left(s_{2}\right)-\eta\left(s_{1}\right)\right| \\
& +\frac{\operatorname{Lip}(f)^{p_{*}}}{p_{*}}\left|r_{2}-r_{1}\right|+\operatorname{Lip}(f) \int_{\xi\left(t_{1}\right)}^{\xi\left(t_{2}\right)} \frac{d t}{b(t)}
\end{aligned}
$$

Here we used the fact that local Lipschitz constant is an upper gradient in the first inequality. The second inequality follows from (2.6), (3.4) and (3.5). The third inequality follows from (2.6) and (3.4) again. Therefore $(r, s, t) \mapsto \int_{M} P_{\xi(t)} Q_{r} f d \mu(\eta(s))$ is continuous on $[0, h] \times\left[t^{\prime}, t^{\prime \prime}\right]^{2}$ and locally Lipschitz on $[0, h] \times\left[t^{\prime}, t^{\prime \prime}\right] \times\left(t^{\prime}, t^{\prime \prime}\right]$. In particular, $r \mapsto$ 
$\int_{M} P_{\xi\left(r+t^{\prime}\right)} Q_{r} f d \mu\left(\eta\left(r+t^{\prime}\right)\right)$ is continuous on $[0, h]$ and locally Lipschitz on $(0, h]$. Hence we can apply [5, Lemma 4.3.4] twice to obtain

$$
\begin{array}{r}
\int_{M} P_{\xi\left(t^{\prime \prime}\right)} Q_{h} f d \mu\left(\eta\left(t^{\prime \prime}\right)\right)-\int_{M} P_{\xi\left(t^{\prime}\right)} f d \mu\left(\eta\left(t^{\prime}\right)\right)=\int_{t^{\prime}}^{t^{\prime \prime}} \frac{\partial}{\partial r}\left(\int_{M} P_{\xi(r)} Q_{r-t^{\prime}} f d \mu(\eta(r))\right) d r \\
\leq \int_{t^{\prime}}^{t^{\prime \prime}} \int_{\operatorname{Geo}(M)}\left(\left|\nabla P_{\xi(r)} Q_{r-t^{\prime}} f\right|(\gamma(\eta(r)))|\dot{\gamma}|(\eta(r)) \eta^{\prime}(r)+\xi^{\prime}(r) \mathscr{L} P_{\xi(r)} Q_{r-t^{\prime}} f(\gamma(\eta(r)))\right. \\
\left.-\frac{1}{p_{*}} P_{\xi(r)}\left(\left|\nabla Q_{r-t^{\prime}} f\right|^{p_{*}}\right)(\gamma(\eta(r)))\right) \Gamma(d \gamma) d r
\end{array}
$$

with the aid of (3.6). By the Hölder inequality and (2.6),

$$
\begin{aligned}
\mid \nabla & P_{\xi(r)} Q_{r-t^{\prime}} f|(\gamma(r))| \dot{\gamma} \mid(\eta(r)) \eta^{\prime}(r)+\xi^{\prime}(r) \mathscr{L} P_{\xi(r)} Q_{r-t^{\prime}} f(\gamma(r)) \\
\leq & \left(d(\gamma(0), \gamma(1))^{\beta} \eta^{\prime}(r)^{\beta}+\left(\frac{\xi^{\prime}(r)}{a(\xi(r)) b(\xi(r))}\right)^{\beta}\right)^{1 / \beta} \\
& \times\left(\left|\nabla P_{\xi(r)} Q_{r-t^{\prime}} f\right|(\gamma(r))^{\beta_{*}}+a(\xi(r))^{\beta *} b(\xi(r))^{\beta_{*}}\left|\mathscr{L} P_{\xi(r)} Q_{r-t^{\prime}} f(\gamma(r))\right|^{\beta_{*}}\right)^{1 / \beta_{*}} \\
\leq & \left(a(\xi(r))^{\beta} d(\gamma(0), \gamma(1))^{\beta} \eta^{\prime}(r)^{\beta}+\left(\frac{\xi^{\prime}(r)}{b(\xi(r))}\right)^{\beta}\right)^{1 / \beta} P_{\xi(r)}\left(\left|\nabla Q_{r-t^{\prime}} f\right|^{p_{*}}\right)(\gamma(r))^{1 / p_{*}} .
\end{aligned}
$$

By combining the last inequality with (3.16) and (3.14), we obtain

$$
\begin{aligned}
& \frac{1}{p}\left(\frac{W_{p}\left(P_{\xi\left(t^{\prime}\right)}^{*} \mu\left(\eta\left(t^{\prime}\right)\right), P_{\xi\left(t^{\prime \prime}\right)}^{*} \mu\left(\eta\left(t^{\prime \prime}\right)\right)\right)}{h}\right)^{p} \\
& \quad \leq \frac{1}{p h} \int_{t^{\prime}}^{t^{\prime \prime}} \int_{\mathrm{Geo}(M)}\left(a(\xi(r))^{\beta} d(\gamma(0), \gamma(1))^{\beta} \eta^{\prime}(r)^{\beta}+\left(\frac{\xi^{\prime}(r)}{b(\xi(r))}\right)^{\beta}\right)^{p / \beta} \Gamma(d \gamma) d r
\end{aligned}
$$

by the Hausdorff-Young inequality. This estimate yields that $r \mapsto P_{\xi(r)}^{*} \mu(\eta(r))$ is an absolutely continuous curve in $W_{p}$ and its metric derivative, denoted by $\left|\partial_{r}\left(P_{\xi(r)}^{*} \mu(\eta(r))\right)\right|_{W_{p}}$, satisfies

$$
\left|\partial_{r}\left(P_{\xi(r)}^{*}(\eta(r))\right)\right|_{W_{p}}^{p} \leq \int_{\operatorname{Geo}(M)}\left(a(\xi(r))^{\beta} d(\gamma(0), \gamma(1))^{\beta} \eta^{\prime}(r)^{\beta}+\left(\frac{\xi^{\prime}(r)}{b(\xi(r))}\right)^{\beta}\right)^{p / \beta} \Gamma(d \gamma)
$$

for almost every $r \in[0,1]$. Then the conclusion follows if both $\mu_{0}$ and $\mu_{1}$ are Dirac measures by using the property of metric derivative reviewed in Section 2. Indeed, in this case $\Gamma$ is a Dirac measure on a minimal geodesic joining points where Dirac masses are taking place and thus $p$-th powers in the both side of (3.18) can be removed. As remarked at the beginning of the proof, it is sufficient to complete the proof.

Proposition 3.6 Assume (A2), (A3) and (2) ae $^{*}$. Let $\eta$ and $\xi$ be as in Proposition 3.5. Then, (3.13) holds for $\mu_{0}, \mu_{1} \in \mathscr{P}(M)$. 
Proof. We closely follow the proof of Proposition 3.5. We first show (3.17) when $\mu_{0}, \mu_{1} \in \mathscr{P}(M)$ have bounded supports and bounded densities with respect to $\mathfrak{m}$. Let $\Gamma$ be a dynamic optimal coupling associated with a geodesics $(\mu(r))_{r \in[0,1]}$ from $\mu_{0}$ to $\mu_{1}$ given as in (A3). In the Kantorovich duality (3.14), we may restrict the class of test functions $f$ to be $C^{\operatorname{Lip}}(M)$ with bounded supports. For such $f, Q_{r} f$ belongs to the same class again for any $r>0$. In particular, the local finiteness of $\mathfrak{m}$ implies $Q_{r} f \in L^{q}(\mathfrak{m})$. Thus the combination of (A2) and the choice of $(\mu(r))_{r \in[0,1]}$ make the computation in (3.15) and (3.16) valid. Indeed, though (2.6) holds only $\mathfrak{m}$-a.e., it is sufficient in this case since $\mu(r) \ll \mathfrak{m}$ by $(\mathrm{A} 3)$. Then the rest of the proof of Proposition 3.5 works in exactly the same way.

Next we take an approximation of Dirac measures. By applying (3.17) with $t^{\prime}=t^{\prime \prime}=t$ and $\eta(r)=r$, we obtain

$$
W_{p}\left(P_{t}^{*} \mu_{0}, P_{t}^{*} \mu_{1}\right) \leq a(t) W_{p}\left(\mu_{0}, \mu_{1}\right) .
$$

By virtue of this estimate, (3.13) for Dirac measures follows by tending $\mu_{i} \rightarrow \delta_{x_{i}}$ with respect to $W_{p}$ in $(3.17)$ for each $x_{0}, x_{1} \in \mathscr{P}(M)$.

Now we will show the implication " $(2)^{*} \Rightarrow(1)$ " in Theorem 2.1 and the corresponding implication " $(2)_{\mathrm{ae}}^{*} \Rightarrow(\mathbf{1})$ " in Theorem 2.2 . We give the proof only to the former one since the other proof goes in exactly the same way. We apply Proposition 3.5 with specified $\xi$ and $\eta$. Let us define $\Xi:[s, t] \rightarrow[0, \infty)$ by $\Xi(r):=J([s, r])$, where $J$ is given in the statement of Theorem 2.1. By using it, we choose $\xi$ and $\eta$ as follows:

$$
\begin{aligned}
& \xi(r):=\Xi^{-1}((\Xi(t)-\Xi(s)) r+\Xi(s)), \\
& \eta(r):=\left(\int_{0}^{1} \frac{d u}{a(\xi(u))}\right)^{-1} \int_{0}^{r} \frac{d u}{a(\xi(u))} .
\end{aligned}
$$

We can easily verify that $\xi$ and $\eta$ satisfy all conditions we supposed in Proposition 3.5. Moreover, we have

$$
\begin{aligned}
\frac{\xi^{\prime}(r)}{b(\xi(r))} & =(\Xi \circ \xi)^{\prime}(r) \equiv \Xi(t)-\Xi(s)=J([s, t]) \\
a(\xi(r)) \eta^{\prime}(r) & \equiv\left(\int_{0}^{1} \frac{d u}{a(\xi(u))}\right)^{-1}=\left(\frac{1}{J([s, t])} \int_{0}^{1} \frac{\xi^{\prime}(u) d u}{a(\xi(u)) b(\xi(u))}\right)^{-1} \\
& =\left(\frac{1}{J([s, t])} \int_{s}^{t} \frac{J(d r)}{a(r)}\right)^{-1} .
\end{aligned}
$$

By substituting them into (3.13), we obtain the desired inequality (2.5).

By putting our arguments together, we complete the proof of Theorem 2.1, Theorem 2.2 and Corollary 2.3.

Remark 3.7 The combination of Proposition 3.1 and Theorem 2.1 (or Theorem 2.2) implies that the inequality (3.7) has a self-improvement property. That is, starting from a less sharp estimate of type (3.7), we can obtain a sharper estimate of type (2.5) by passing through (2.6). 
On the other hand, we can easily obtain many weaker inequalities of type (3.7) from (2.6). Indeed, since our proof is based on Proposition 3.5 and an appropriate choice of space-time reparametrization $\eta$ and $\xi$, a bad choice of $\eta$ and $\xi$ produces a weaker estimate. Nevertheless, Proposition 3.1 ensures that such a weaker estimate reproduces (2.6) and consequently such a weaker estimate of type (3.7) can be equivalent to (2.6). Indeed, our choice of $\xi$ and $\eta$ in the proof of Theorem 2.1 may not be optimal. $\xi$ is a minimizer of the right hand side of (3.13) when $\eta^{\prime} \equiv 0$ and $\eta$ is a minimizer of the same quantity for fixed $\xi$. Even in the case of Corollary 2.4, the genuine minimizer $(\xi, \eta)$ seems to be rather complicated (cf. Remark 3.8).

By using Proposition 3.5, or Proposition 3.6, we also conclude Theorem 2.5.

Proof of Theorem 2.5. We only give the proof of the implication "(1.2) $\Rightarrow(2.8)$ " in (i) since the other implication is already shown at the end of Section 3.2 and the corresponding assertion in (ii) can be shown in the same way by using Proposition 3.6 instead of Proposition 3.5. To complete the proof it is sufficient to obtain the following differential inequality: For $u>0$ and $\lambda \geq 1$,

$$
\begin{aligned}
\frac{\partial}{\partial u} \mathfrak{s}_{K / N}\left(\frac{W_{2}\left(P_{\lambda^{-1} u}^{*} \mu, P_{\lambda u}^{*} \nu\right)}{2}\right)^{2} & \\
& \leq-K\left(\lambda+\lambda^{-1}\right) \mathfrak{s}_{K / N}\left(\frac{W_{2}\left(P_{\lambda^{-1} u}^{*} \mu, P_{\lambda u}^{*} \nu\right)}{2}\right)^{2}+\frac{N}{2}\left(\lambda+\lambda^{-1}-2\right)
\end{aligned}
$$

Indeed, letting $\lambda=\sqrt{t / s}$ and $r=\sqrt{t s}$ and applying the Gronwall lemma to give an estimate of $\mathfrak{s}_{K / N}\left(W_{2}\left(P_{\lambda^{-1} r}^{*} \mu, P_{\lambda r}^{*} \nu\right) / 2\right)^{2}$ yield the conclusion. In the sequel, we use the abbreviation $w:=W_{2}\left(P_{\lambda^{-1} u}^{*} \mu, P_{\lambda u}^{*} \nu\right)$ for simplicity of notation. For $h>0$, let us define $l$, $\theta_{h}$ and $\xi_{h}$ as follows:

$$
\begin{aligned}
& l(r):= \begin{cases}\mathfrak{c}_{K / N}^{-1}\left(\mathrm{e}^{-K r}\right) & (K \neq 0), \\
\sqrt{2 N r} & (K=0),\end{cases} \\
& \theta_{h}(r):= \begin{cases}\frac{l(\lambda h) \mathfrak{s}_{K / N}(w r)+l\left(\lambda^{-1} h\right) \mathfrak{s}_{K / N}(w(1-r))}{\mathfrak{s}_{K / N}(w)} & (K \neq 0, w \neq 0), \\
l(\lambda h) r+l\left(\lambda^{-1} h\right)(1-r) & (K=0 \text { or } w=0),\end{cases} \\
& \xi_{h}(r):=l^{-1}\left(\theta_{h}(r)\right)= \begin{cases}-\frac{1}{K} \log \left(\mathfrak{c}_{K / N}\left(\theta_{h}(r)\right)\right) & (K \neq 0), \\
\frac{\theta_{h}(r)^{2}}{2 N} & (K=0) .\end{cases}
\end{aligned}
$$

In what follows, we only consider the case $K \neq 0$ and $w \neq 0$ for simplicity of presentation. Indeed, the same argument also works in other cases. Note that $\xi_{h}$ is $C^{1}$-increasing surjection from $[0,1]$ to $\left[\lambda^{-1} h, \lambda h\right]$. Thus applying Proposition 3.5 with $a(t)=\mathrm{e}^{-K t}$, $b(r)=\sqrt{\left(\mathrm{e}^{2 K t}-1\right) /(N K)}, t^{\prime}=\lambda^{-1} h, t^{\prime \prime}=\lambda h, \mu_{0}=P_{\lambda^{-1} u}^{*} \mu, \mu_{1}=P_{\lambda u}^{*} \nu, \eta(r)=r$ and $\xi=\xi_{h}$, we obtain

$$
W_{2}\left(P_{\lambda^{-1}(u+h)}^{*} \mu, P_{\lambda(u+h)}^{*} \nu\right)^{2} \leq \int_{0}^{1}\left(\mathrm{e}^{-2 K \xi_{h}(r)} w^{2}+\frac{N K}{\mathrm{e}^{2 K \xi_{h}(r)}-1} \xi_{h}^{\prime}(r)^{2}\right) d r .
$$


Since we have

$$
\xi_{h}^{\prime}(r)=\frac{\mathfrak{t}_{K / N}\left(\theta_{h}(r)\right)}{N} \theta_{h}^{\prime}(r)=b\left(\xi_{h}(r)\right) \theta_{h}^{\prime}(r), \quad \lim _{h \rightarrow 0} \frac{l(\alpha h)}{\sqrt{h}}=\sqrt{2 N \alpha}
$$

for $\alpha \geq 0$ and the addition formulae

$$
\begin{aligned}
\mathfrak{c}_{K / N}(u+v) & =\mathfrak{c}_{K / N}(u) \mathfrak{c}_{K / N}(v)-\frac{K}{N} \mathfrak{s}_{K / N}(u) \mathfrak{s}_{K / N}(v), \\
\mathfrak{s}_{K / N}(u+v) & =\mathfrak{s}_{K / N}(u) \mathfrak{c}_{K / N}(v)+\mathfrak{c}_{K / N}(u) \mathfrak{s}_{K / N}(v),
\end{aligned}
$$

(3.20) implies

$$
\begin{gathered}
\frac{\partial}{\partial u} W_{2}\left(P_{\lambda^{-1} u}^{*} \mu, P_{\lambda u}^{*} \nu\right)^{2} \leq \limsup _{h \rightarrow 0} \frac{1}{h} \int_{0}^{1}\left(\left(\mathrm{e}^{-2 K \xi_{h}(r)}-1\right) w^{2}+\theta_{h}^{\prime}(r)^{2}\right) d r \\
=\int_{0}^{1}\left\{-2 K w^{2}\left(\frac{\sqrt{\lambda} \mathfrak{s}_{K / N}(w r)+\sqrt{\lambda^{-1}} \mathfrak{s}_{K / N}(w(1-r))}{\mathfrak{s}_{K / N}(w)}\right)^{2}\right. \\
\left.+2 N w^{2}\left(\frac{\sqrt{\lambda} \mathfrak{c}_{K / N}(w r)-\sqrt{\lambda^{-1}} \mathfrak{c}_{K / N}(w(1-r))}{\mathfrak{s}_{K / N}(w)}\right)^{2}\right\} d r \\
=\frac{2 N w^{2}}{\mathfrak{s}_{K / N}(w)^{2}}\left\{\left(\lambda+\lambda^{-1}\right) \int_{0}^{1} \mathfrak{c}_{K / N}(2 w r) d r-2 \int_{0}^{1} \mathfrak{c}_{K / N}(w(2 r-1)) d r\right\} \\
=\frac{4 w}{\mathfrak{s}_{K / N}(w)}\left(-K\left(\lambda+\lambda^{-1}\right) \mathfrak{s}_{K / N}\left(\frac{w}{2}\right)^{2}+\frac{N}{2}\left(\lambda+\lambda^{-1}-2\right)\right)
\end{gathered}
$$

Since

$$
\frac{\partial}{\partial u} \mathfrak{s}_{K / N}\left(\frac{1}{2} W_{2}\left(P_{\lambda^{-1} u}^{*} \mu, P_{\lambda u}^{*} \nu\right)\right)^{2}=\frac{\mathfrak{s}_{K / N}(w)}{4 w} \frac{\partial}{\partial u} W_{2}\left(P_{\lambda^{-1} u}^{*} \mu, P_{\lambda u}^{*} \nu\right)^{2},
$$

(3.21) immediately yields (3.19).

Remark 3.8 The time parametrization $\xi_{h}$ in the proof of Theorem 2.5 is nearly optimal. Indeed, a minimizer $\xi$ of the right hand side of (3.13) under the specified choice of $a, b$ and $\eta(r)=r$, as in the proof of Theorem 2.5, is a solution to an ordinary differential equation. It can be expressed in the following simple form:

$$
\begin{aligned}
\xi(r) & =l^{-1}(\theta(r)), \\
\theta^{\prime \prime}(r) & =-\frac{K w^{2}}{2 N} \mathfrak{s}_{K / N}(2 \theta(r)), \\
\xi(0) & =s, \xi(1)=t .
\end{aligned}
$$

However, the solution $\theta$ becomes an elliptic function in general. To avoid technical difficulties, we have considered comparison functions instead by linearizing the equation for $\theta$ since we are only interested in the case $s, t \ll 1$ in our argument and then $\theta$ must be small also. As a result, we obtain $\theta_{h}$ in the proof of Theorem 2.5. 


\section{A Coupling method on Riemannian manifolds}

In this section, we are supposed to be in the framework of Theorem 2.6. In particular, $M$ is an $m$-dimensional complete Riemannian manifold and $P_{t}$ is given by the integral operator associated with the distribution of the diffusion process generated by $\mathscr{L}=\Delta+Z$. Note that $X(t)$ is conservative under $(2.9)$ (see $[26,35]$ for instance) and hence $P_{t}$ defines a Markov kernel. Since we are on a smooth space, (A1) is satisfied.

In the following argument, we always assume $\operatorname{diam}(M)<\sqrt{(N-1) \pi / K}$ when $K>0$ to avoid the singularity when $d(x, y)=\sqrt{(N-1) \pi / K}$ (see Remark 4.1 below).

Remark 4.1 When $K>0$, it is known that (2.9) yields $\operatorname{diam}(M) \leq \sqrt{(N-1) \pi / K}$ (see [27]). Thus the above assumption only exclude the case $\operatorname{diam}(M)=\sqrt{(N-1) \pi / K}$. Even when it is the case, we can prove the same conclusion for $K^{\prime}<K$ in (2.9) instead of $K$ and finally let $K^{\prime} \uparrow K$ to obtain the full statement from the one involving $K^{\prime}$. As a matter of fact, $\operatorname{diam}(M)=\sqrt{(N-1) \pi / K}$ happens only when $N=\operatorname{dim} M, Z \equiv 0$ and $M$ is isometric to the sphere of the constant sectional curvature $K /(N-1)$ (see [27]).

\subsection{The Case under the ABsence of the CUt Locus}

In what follows, we explain how our coupling method works. For this purpose, we assume that the cut locus of $M$ is empty and $Z=0$ in this section to avoid technical difficulties. In this case, we can construct a coupling of Brownian motions on $M$ directly by solving a coupled SDE. We refer to [22] for basic notions in this section.

Let $\mathcal{O}(M)$ be the orthonormal frame bundle of $M$ and $\pi$ a canonical projection $\mathcal{O}(M) \rightarrow M$. Fix $\tau_{2}, \tau_{1}>0$ and $x, y \in M$ with $x \neq y$ for a while. Let us consider a coupling of (time-scaled) horizontal diffusion processes $\left(U_{1}(t), U_{2}(t)\right)$ by parallel transport. To define them, we first prepare some notations. For $i=1, \ldots, n$, let $H_{i}$ be a canonical horizontal vector field on $\mathcal{O}(M)$. That is, $H_{i}(u)$ is the horizontal lift (associated with the Levi-Civita connection) of $u e_{i}$, where $\left(e_{i}\right)_{i=1}^{n}$ is the canonical basis of $\mathbb{R}^{n}$. Let $\tilde{H}_{i}: \mathcal{O}(M) \times \mathcal{O}(M) \rightarrow T \mathcal{O}(M)$ be a horizontal vector field coupled with $\left(H_{i}\right)_{i=1}^{n}$ as follows: For $u_{1}, u_{2} \in \mathcal{O}(M), \tilde{H}_{i}\left(u_{1}, u_{2}\right)$ is the horizontal lift of $/ / \pi\left(u_{1}\right) \pi\left(u_{2}\right) u_{1} e_{i}$, where $/ / z w$ is the parallel transport of tangent vectors from $T_{z} M$ to $T_{w} M$ along a minimal geodesic joining $z$ and $w$ (such a geodesic is unique under the absence of the cut locus). Let $\mathbf{W}(t)=\left(W^{i}(t)\right)_{i=1}^{n}$ be a Brownian motion on $\mathbb{R}^{n}$. Take $u_{1}, u_{2} \in \mathcal{O}(M)$ so that $\pi\left(u_{1}\right)=x$, $\pi\left(u_{2}\right)=y$ and $u_{2}=/ / x y u_{1}$. Now we are ready to define $\left(U_{1}(t), U_{2}(t)\right)$. They are defined as a solution to the following system of stochastic differential equations:

$$
\begin{array}{ll}
d U_{1}(t)=\sqrt{2 \tau_{1}} \sum_{i=1}^{n} H_{i}\left(U_{1}(t)\right) \circ d W^{i}(t), & U_{1}(0)=u_{1}, \\
d U_{2}(t)=\sqrt{2 \tau_{2}} \sum_{i=1}^{n} \tilde{H}_{i}\left(U_{1}(t), U_{2}(t)\right) \circ d W^{i}(t), & U_{2}(0)=u_{2} .
\end{array}
$$

Let $X_{i}(t):=\pi\left(U_{i}(t)\right)$. Then $\mathbf{X}(t)=\left(X_{1}(t), X_{2}(t)\right)$ is a coupling by parallel transport of two (time-scaled) Brownian motions. 
Intuitively, infinitesimal motions $d X_{1}(t)$ and $d X_{2}(t)$, which can be regarded as a random element in $T_{X_{1}(t)} M$ and $T_{X_{2}(t)} M$ respectively, are given by scaled "white noises" $\sqrt{2 \tau_{1}} U_{1}(t) \circ d \mathbf{W}(t)$ and $\sqrt{2 \tau_{2}} U_{2}(t) \circ d \mathbf{W}(t)$ respectively, and the second noise is given by the parallel transport of the first noise: $U_{2}(t) \circ d \mathbf{W}(t)=/ / X_{1}(t) X_{2}(t) U_{1}(t) \circ d \mathbf{W}(t)$.

Let us turn to the proof. Let $\rho(t):=d(\mathbf{X}(t))$. By the Itô formula, we obtain

$$
\begin{aligned}
& d \rho(s)^{p}=p \rho(s)^{p-1} \sum_{i=1}^{n}\left(\sqrt{2 \tau_{1}} U_{1}(s) e_{i} \oplus \sqrt{2 \tau_{2}} U_{2}(s) e_{i}\right) d(\mathbf{X}(s)) d W^{i}(s) \\
& +p \rho(s)^{p-1} \sum_{i=1}^{n}\left(\sqrt{\tau_{1}} U_{1}(s) e_{i} \oplus \sqrt{\tau_{2}} U_{2}(s) e_{i}\right)^{2} d(\mathbf{X}(s)) d s \\
& +p(p-1) \rho(s)^{p-2} \sum_{i=1}^{n}\left\{\left(\sqrt{\tau_{1}} U_{1}(s) e_{i} \oplus \sqrt{\tau_{2}} U_{2}(s) e_{i}\right) d(\mathbf{X}(s))\right\}^{2} d s
\end{aligned}
$$

We take an expectation of the integral form of (4.1). To be precise, we must take care on the integrability, but we always assume it in this section for simpler explanation. The expectation of the first term in the right hand side is zero since it is stochastic integral. For the third term, by the first variation formula of arclength, we obtain

$$
\begin{aligned}
\sum_{i=1}^{n} & \left\{\left(\sqrt{\tau_{1}} U_{1}(s) e_{i} \oplus \sqrt{\tau_{2}} U_{2}(s) e_{i}\right) d(\mathbf{X}(s))\right\}^{2} \\
& =\left(\sqrt{\tau_{1}}-\sqrt{\tau_{2}}\right)^{2} \sum_{i=1}^{n}\left\{\nabla_{U_{1}(s) e_{i}} d\left(\cdot, X_{2}(s)\right)\left(X_{1}(s)\right)\right\}^{2} \\
& =\left(\sqrt{\tau_{1}}-\sqrt{\tau_{2}}\right)^{2} .
\end{aligned}
$$

For the second term, the second variation formula of arclength, we obtain

$$
\sum_{i=1}^{n}\left(\sqrt{\tau_{1}} U_{1}(s) e_{i} \oplus \sqrt{\tau_{2}} U_{2}(s) e_{i}\right) d(\mathbf{X}(s))=\sum_{i=1}^{n} I_{\mathbf{X}(s)}\left(\tilde{U}_{\mathbf{X}(s)}^{(i)}(s), \tilde{U}_{\mathbf{X}(s)}^{(i)}(s)\right) d s,
$$

where $I_{z w}$ is the index form along a constant speed minimal geodesic from $z$ to $w$ and $\tilde{U}_{z w}^{(i)}(s)$ is the Jacobi field along the same minimal geodesic whose the boundary values are $\sqrt{\tau_{1}} U_{1}(s) e_{i}$ and $\sqrt{\tau_{2}} U_{2}(s) e_{i}$ respectively. For an upper bound of the index form, we introduce some notations. Set $K^{*}=K /(N-1)$. We define $\Psi=\Psi_{\tau_{1}, \tau_{2}}:(0, \infty) \rightarrow \mathbb{R}$ as follows:

$$
\Psi_{\tau_{1}, \tau_{2}}(r):=(N-1)\left(\frac{\tau_{1}+\tau_{2}}{\mathfrak{t}_{K^{*}}(r)}-\frac{2 \sqrt{\tau_{1} \tau_{2}}}{\mathfrak{s}_{K^{*}}(r)}\right) .
$$

Lemma 4.2 Suppose $N=m$. Let $x, y \in M$ and $\left(f_{i}\right)_{i=1}^{n}$ an orthonormal basis of $T_{x} M$. Then we have

$$
\sum_{i=1}^{n} I_{x y}\left(\tilde{V}_{x y}^{(i)}, \tilde{V}_{x y}^{(i)}\right) \leq \Psi_{\tau_{1}, \tau_{2}}(d(x, y))
$$

where $\tilde{V}_{x y}^{(i)}$ is the Jacobi field along the minimal geodesic joining $x$ and $y$ whose boundary values are $\sqrt{\tau_{1}} f_{i}$ and $\sqrt{\tau_{2}} / / x y f_{i}$ respectively. 
Proof. In the proof, we denote $d(x, y)$ by $d$. Recall that the condition (2.9) is reduced to Ric $\geq K$ in the present case. Let us define $\varphi_{d}:[0, d] \rightarrow \mathbb{R}$ by

$$
\varphi_{d}(u):=\sqrt{\tau_{2}} \frac{\mathfrak{s}_{K^{*}}(u)}{\mathfrak{s}_{K^{*}}(d)}+\sqrt{\tau_{1}} \frac{\mathfrak{s}_{K^{*}}(d-u)}{\mathfrak{s}_{K^{*}}(d)} .
$$

Let us denote the vector field along $\gamma$ given by the parallel transport of $f_{i}$ by $V_{i}(\cdot)$. Then $\varphi_{d}(0) V_{i}(0)=\sqrt{\tau_{1}} f_{i}$ and $\varphi_{d}(d) V_{i}(d)=\sqrt{\tau_{2}} / / x y f_{i}$. Thus the index lemma together with (2.9) yields

$$
\begin{aligned}
\sum_{i=1}^{n} I_{x y}\left(V_{x y}^{(i)}, V_{x y}^{(i)}\right) & \leq(m-1) \int_{0}^{d} \varphi_{d}^{\prime}(u)^{2} d u-K \int_{0}^{d} \varphi_{d}(u)^{2} d u \\
& =(m-1) \int_{0}^{d}\left(\varphi_{d}^{\prime}(u) \varphi_{d}(u)\right)^{\prime} d u \\
& =(m-1)\left(\varphi_{d}^{\prime}(d) \varphi_{d}(d)-\varphi_{d}^{\prime}(0) \varphi_{d}(0)\right) \\
& =(m-1)\left\{\frac{\sqrt{\tau_{2}} \mathfrak{c}_{K^{*}}(d)-\sqrt{\tau_{1}}}{\mathfrak{s}_{K^{*}}(d)} \sqrt{\tau_{2}}-\frac{\sqrt{\tau_{2}}-\sqrt{\tau_{1}} \mathfrak{c}_{K^{*}}(d)}{\mathfrak{s}_{K^{*}}(d)} \sqrt{\tau_{1}}\right\} \\
& =(m-1)\left(\frac{\tau_{1}+\tau_{2}}{\mathfrak{t}_{K^{*}}(d)}-\frac{2 \sqrt{\tau_{1} \tau_{2}}}{\mathfrak{s}_{K^{*}}(d)}\right) .
\end{aligned}
$$

This is nothing but the claim.

Lemma 4.3 For $\tau_{1}, \tau_{2}>0$,

$$
\Psi_{\tau_{1}, \tau_{2}}(r) \leq \begin{cases}-\sqrt{\tau_{1} \tau_{2}} K r+\frac{(N-1)\left(\sqrt{\tau_{2}}-\sqrt{\tau_{1}}\right)^{2}}{r} & (K \geq 0), \\ -\frac{\tau_{1}+\tau_{2}}{2} K r+\frac{(N-1)\left(\sqrt{\tau_{2}}-\sqrt{\tau_{1}}\right)^{2}}{r} & (K<0) .\end{cases}
$$

Proof. By an easy rearrangement, we have

$$
\Psi_{\tau_{1}, \tau_{2}}(r)=(N-1)\left(2 \sqrt{\tau_{1} \tau_{2}} \frac{\mathfrak{c}_{K^{*}}(r)-1}{\mathfrak{s}_{K^{*}}(r)}+\left(\sqrt{\tau_{2}}-\sqrt{\tau_{1}}\right)^{2} \frac{1}{\mathfrak{t}_{K^{*}}(r)}\right) .
$$

When $K \geq 0$,

$$
\frac{\mathfrak{c}_{K^{*}}(r)-1}{\mathfrak{s}_{K^{*}}(r)}=-\sqrt{K^{*}} \tan \left(\frac{\sqrt{K^{*} r}}{2}\right) \leq-\frac{K^{*} r}{2}, \quad \frac{1}{\mathfrak{t}_{K^{*}}(r)} \leq \frac{1}{r} .
$$

By plugging them in (4.6), we obtain the first inequality. Similarly, by a rearrangement,

$$
\Psi_{\tau_{1}, \tau_{2}}(r)=(N-1)\left(\left(\tau_{1}+\tau_{2}\right) \frac{\mathfrak{c}_{K^{*}}(r)-1}{\mathfrak{s}_{K^{*}}(r)}+\left(\sqrt{\tau_{2}}-\sqrt{\tau_{1}}\right)^{2} \frac{1}{\mathfrak{s}_{K^{*}}(r)}\right) .
$$

When $K<0$,

$$
\frac{\mathfrak{c}_{K^{*}}(r)-1}{\mathfrak{s}_{K^{*}}(r)}=\sqrt{-K^{*}} \tanh \left(\frac{\sqrt{-K^{*}} r}{2}\right) \leq-\frac{K^{*} r}{2}, \quad \frac{1}{\mathfrak{s}_{K^{*}}(r)} \leq \frac{1}{r} .
$$

and the second inequality follows by plugging them in (4.7). 
Let us define $\tau^{*}$ by

$$
\tau^{*}:= \begin{cases}\sqrt{\tau_{1} \tau_{2}} & (K \geq 0) \\ \frac{\tau_{1}+\tau_{2}}{2} & (K<0) .\end{cases}
$$

By using (4.2), (4.3), Lemma 4.2 and Lemma 4.3 to give an estimate of the expectation of the integral of (4.1) in time from $u>0$ to $u^{\prime}>u$, when $N=m$, we obtain

$$
\begin{aligned}
\mathbb{E}\left[\rho\left(u^{\prime}\right)^{p}\right] \leq & \mathbb{E}\left[\rho(u)^{p}\right]-p \tau^{*} K \int_{u}^{u^{\prime}} \mathbb{E}\left[\rho(v)^{p}\right] d v \\
& +p(N+p-2) \int_{u}^{u^{\prime}} \mathbb{E}\left[\rho(v)^{p-2}\right] d v\left(\sqrt{\tau_{1}}-\sqrt{\tau_{2}}\right)^{2} \\
\leq & \mathbb{E}\left[\rho(u)^{p}\right]-p \tau^{*} K \int_{u}^{u^{\prime}} \mathbb{E}\left[\rho(v)^{p}\right] d v \\
& +p(N+p-2) \int_{u}^{u^{\prime}} \mathbb{E}\left[\rho(v)^{p}\right]^{(p-2) / p} d v\left(\sqrt{\tau_{1}}-\sqrt{\tau_{2}}\right)^{2} .
\end{aligned}
$$

Obviously, the same is also true even when $N>m$. It yields

$$
\frac{\partial}{\partial u}\left(\mathbb{E}\left[\rho(u)^{p}\right]^{2 / p}\right) \leq-2 \tau^{*} K \mathbb{E}\left[\rho(u)^{p}\right]^{2 / p}+2(N+p-2)\left(\sqrt{\tau_{1}}-\sqrt{\tau_{2}}\right)^{2} .
$$

Thus, by the Gronwall lemma for $\mathbb{E}\left[\rho(t)^{p}\right]^{2 / p}$ as a function of $t$, we obtain

$$
\mathbb{E}\left[\rho(1)^{p}\right]^{2 / p} \leq \mathrm{e}^{-2 K \tau^{*}} \mathbb{E}\left[\rho(0)^{p}\right]^{2 / p}+\frac{(N+p-2)\left(1-\mathrm{e}^{-2 K \tau^{*}}\right)}{K \tau^{*}}\left(\sqrt{\tau_{2}}-\sqrt{\tau_{1}}\right)^{2} .
$$

By the choice of the initial condition, $\mathbb{E}\left[\rho(0)^{2}\right]=d(x, y)^{2}$. Moreover, since the law of $\left(X_{1}(1), X_{2}(1)\right)$ is a coupling of $P_{\tau_{1}}^{*} \delta_{x}$ and $P_{\tau_{2}}^{*} \delta_{y}$, the definition of $L^{p}$-Wasserstein distance implies that

$$
W_{p}\left(P_{\tau_{1}}^{*} \delta_{x}, P_{\tau_{2}}^{*} \delta_{y}\right)^{2} \leq \mathrm{e}^{-2 K \tau^{*}} d(x, y)^{2}+\frac{(N+p-2)\left(1-\mathrm{e}^{-2 K \tau^{*}}\right)}{K \tau^{*}}\left(\sqrt{\tau_{2}}-\sqrt{\tau_{1}}\right)^{2} .
$$

To obtain (2.10) from (4.8), we prepare an $L^{p}$-version of the Bakry-Émery's curvaturedimension condition as well as its connection with (2.11). For $f \in C^{3}(M)$, let us define $\Gamma_{2}(f)$ by

$$
\Gamma_{2}(f)=\frac{1}{2} \mathscr{L}|\nabla f|^{2}-\langle\nabla f, \nabla \mathscr{L} f\rangle
$$

Proposition 4.4 The following conditions are equivalent:

(i) For any $f \in C_{b}^{\mathrm{Lip}}(M)$ and $x \in M$,

$$
\left|\nabla P_{t} f\right|(x)^{2} \leq \mathrm{e}^{-2 K t} P_{t}\left(|\nabla f|^{p_{*}}\right)(x)^{2 / p_{*}}-\frac{1-\mathrm{e}^{-2 K t}}{(N+p-2) K}\left(\mathscr{L} P_{t} f(x)\right)^{2} .
$$


(ii) For each $t>0$, there is a constant $C(t)>0$ satisfying $\lim _{t \downarrow 0} \frac{C(t)}{t}=1$ and

$$
\left|\nabla P_{t} f\right|(x)^{2} \leq \mathrm{e}^{-2 K t} P_{t}\left(|\nabla f|^{p_{*}}\right)(x)^{2 / p_{*}}-\frac{2 C(t)}{N+p-2}\left(\mathscr{L} P_{t} f(x)\right)^{2}
$$

for any $f \in C_{b}^{\mathrm{Lip}}(M)$ and $x \in M$.

(iii) For any $f \in C^{\infty}(M), x \in M$ and $\delta>0$,

$$
\left(|\nabla f|^{2}+\delta\right)\left(\Gamma_{2}(f)(x)-K|\nabla f|(x)^{2}-\frac{(\mathscr{L} f(x))^{2}}{N+p-2}\right) \geq\left.\frac{p-2}{4(p-1)}|\nabla| \nabla f\right|^{2} \mid(x)^{2} .
$$

Proof. The implication "(i) $\Rightarrow$ (ii)" is obvious. For the proof of "(ii) $\Rightarrow$ (iii)", we claim

$$
P_{t}\left((g+\delta)^{r}\right)^{1 / r}-\delta \geq P_{t}\left(g^{r}\right)^{1 / r}
$$

for $r \in(0,1), \delta>0$ and $g: M \rightarrow[0, \infty)$ measurable. For (4.9), it suffices to show $\partial_{\delta} P_{t}\left((g+\delta)^{r}\right)^{1 / r} \geq 1$. Let us take $\alpha>0$ so that $\alpha^{-1}=r^{-1}+(1-r)^{-1}$. Then we have

$$
\begin{aligned}
\partial_{\delta} P_{t}\left((g+\delta)^{r}\right)^{1 / r} & =P_{t}\left((g+\delta)^{r}\right)^{1 / r-1} P_{t}\left((g+\delta)^{r-1}\right) \\
& =\left\{P_{t}\left((g+\delta)^{r}\right)^{\alpha / r} P_{t}\left(\left(\frac{1}{g+\delta}\right)^{1-r}\right)^{\alpha /(1-r)}\right\}^{(1-r) / \alpha} \\
& \geq\left\{P_{t}\left((g+\delta)^{\alpha} \cdot\left(\frac{1}{g+\delta}\right)^{\alpha}\right)\right\}^{(1-r) / \alpha}=1
\end{aligned}
$$

by the Hölder inequality for $P_{t}$. Thus the claim holds.

Suppose that (ii) holds. By applying (4.9) with $g=|\nabla f|^{2}$ and $r=p_{*} / 2$ to (ii),

$$
\left|\nabla P_{t} f\right|^{2} \leq \mathrm{e}^{-2 K t}\left(P_{t}\left(\left(|\nabla f|^{2}+\delta\right)^{p_{*} / 2}\right)^{2 / p_{*}}-\delta\right)-\frac{2 C(t)}{N+p-2}\left(\mathscr{L} P_{t} f\right)^{2} .
$$

Since the equality holds at $t=0$ in the last inequality, by taking a derivative with respect to $t$ at $t=0$, we obtain

$$
2\langle\nabla f, \nabla \mathscr{L} f\rangle \leq \mathscr{L}|\nabla f|^{2}+\frac{p_{*}-2}{2} \cdot \frac{\left.\left.|\nabla| \nabla f\right|^{2}\right|^{2}}{|\nabla f|^{2}+\delta}-\frac{2}{N+p-2}(\mathscr{L} f)^{2}-2 K|\nabla f|^{2} .
$$

By an easy rearrangement, this inequality yields (iii).

We turn to show "(iii) $\Rightarrow$ (i)". To deal with technical difficulties, we consider several bounds on derivatives of $P_{s} f$. By (iii), we have $\Gamma_{2}(f) \geq K|\nabla f|^{2}$. It is well-known as Bakry-Émery theory that it yields Ric $-(\nabla Z)^{b} \geq K$. Then we can obtain (1.3) and hence (1.1) for $f \in C_{b}^{\text {Lip }}(M)$ (see $[25,26]$ for instance). Note that we avoid a standard argument in Bakry-Émery theory and take a detour to obtain (1.1) here in order to take the fact that $Z$ can be of non-gradient type into account. As a result of (1.1), $\sup _{x \in M, t \leq T}\left|\nabla P_{t} f\right|(x)<\infty$ for $f \in C_{b}^{\operatorname{Lip}}(M)$. 
Let $\delta>0$. First we consider the case $f \in C_{0}^{\infty}(M)$. Let us define $F:[0, t] \times M \rightarrow \mathbb{R}$ by

$$
F(s, x)=\left(\left|\nabla P_{t-s} f\right|(x)^{2}+\delta\right)^{p_{*} / 2} .
$$

Note that $F$ is bounded. Recall $p_{*} \geq 2$. To avoid technicalities on integrability, we will employ a stochastic analytic argument. Let $s_{0} \in(0, t)$. By the Itô formula, for $s \in\left[s_{0}, t\right)$,

$$
M^{F}(s):=F(s, X(s))-F\left(s_{0}, X\left(s_{0}\right)\right)-\int_{s_{0}}^{s}\left(\frac{\partial F}{\partial s}(u, X(u))+\mathscr{L} F(u, X(u))\right) d u
$$

is a local martingale. By using (iii), we have

$$
\begin{aligned}
& \frac{\partial F}{\partial s}(s, X(s))+\mathscr{L} F(s, X(s)) \\
& \quad=p_{*}\left(\left|\nabla P_{t-s} f\right|(X(s))^{2}+\delta\right)^{p_{*} / 2-1}\left(\Gamma_{2}\left(P_{t-s} f\right)+\frac{p_{*}-2}{4} \cdot \frac{\left.\left.|\nabla| \nabla P_{t-s} f\right|^{2}\right|^{2}}{\left|\nabla P_{t-s} f\right|^{2}+\delta}\right)(X(s)) \\
& \quad \geq p_{*}\left(\left|\nabla P_{t-s} f\right|(X(s))^{2}+\delta\right)^{p_{*} / 2-1}\left(K\left|\nabla P_{t-s} f\right|^{2}+\frac{\left(P_{t-s}(\mathscr{L} f)\right)^{2}}{N+p-2}\right)(X(s)) .
\end{aligned}
$$

Note that the last term is bounded from below even when $K<0$. Thus, by localizing $M^{F}(s)$, taking expectation and applying the Fatou lemma, we obtain

$$
\begin{aligned}
& \mathbb{E}[F(s, X(s))]-\mathbb{E}\left[F\left(s_{0}, X\left(s_{0}\right)\right)\right] \\
& \geq p_{*} \int_{s_{0}}^{s} \mathbb{E}\left[\left(\left|\nabla P_{t-u} f\right|(X(u))^{2}+\delta\right)^{p_{*} / 2-1}\left(K\left|\nabla P_{t-u} f\right|^{2}+\frac{\left(P_{t-u}(\mathscr{L} f)\right)^{2}}{N+p-2}\right)(X(u))\right] d s \\
& \geq p_{*} \int_{s_{0}}^{s}\left\{K \mathbb{E}[F(u, X(u))]+\frac{\left(\mathscr{L} P_{t} f\right)(x)^{2}}{(N+p-2) \mathbb{E}\left[\left(\left|\nabla P_{t-u} f\right|(X(u))^{2}+\delta\right)^{1-p_{*} / 2}\right]}\right. \\
& \left.\quad-K \delta \mathbb{E}\left[\left(\left|\nabla P_{t-u} f\right|(X(u))^{2}+\delta\right)^{p_{*} / 2-1}\right]\right\} d u \\
& \geq p_{*} \int_{s_{0}}^{s}\left\{K \mathbb{E}[F(u, X(u))]+\frac{\left(\mathscr{L} P_{t} f\right)(x)^{2}}{N+p-2} \mathbb{E}\left[\left(\left|\nabla P_{t-u} f\right|(X(u))^{2}+\delta\right)^{p_{*} / 2}\right]^{1-2 / p_{*}}\right. \\
& \left.-K \delta^{p_{*} / 2}\right\} d u .
\end{aligned}
$$

Here we have used the Schwarz inequality and $P_{t-s} \mathscr{L}=\mathscr{L} P_{t-s}$ in the second inequality and the Hölder inequality in the third inequality. Note that $\mathbb{E}[F(u, X(u))]$ is strictly positive and (uniformly) continuous in $u \in[0, t]$. Thus, by virtue of the mean value theorem for $r \mapsto r^{2 / p_{*}}$ and the last inequality, for $\varepsilon>0$ there is a constant and $\eta>0$ being independent of $s_{0}$ and $s$ such that

$$
\begin{aligned}
\mathrm{e}^{-2 K s} \mathbb{E}[F(s, X(s))]^{2 / p_{*}}-\mathrm{e}^{-2 K s_{0}} \mathbb{E} & {\left[F\left(s_{0}, X\left(s_{0}\right)\right)\right]^{2 / p_{*}} } \\
& \geq\left(\frac{\left(\mathscr{L} P_{t} f\right)(x)^{2}}{N+p-2}-K \delta^{p_{*} / 2}\right) \int_{s_{0}}^{s} \mathrm{e}^{-2 K r} d r-\varepsilon\left(s-s_{0}\right)
\end{aligned}
$$


whenever $\left|s-s_{0}\right|<\eta$. By taking a sum and a limit, we extend the last estimate for $s, s_{0} \in[0, t]$ with $s>s_{0}$. Then the desired estimate holds by substituting $s_{0}=0$ and $s=t, \varepsilon \downarrow 0$ and $\delta \downarrow 0$.

Finally we will show (i) for $f \in C_{b}^{\mathrm{Lip}}(M)$. The first step is to rewrite (i) in integral form as in the condition (3). For $x, y \in M$ with $x \neq y$, let $\gamma:[0,1] \rightarrow M$ be a minimal geodesic from $x$ to $y$. Then, for $f \in C_{0}^{\infty}(M), t \geq s>0$ and $\xi(r)=r t+(1-r) s$, we have

$$
\begin{aligned}
\mid P_{t} f(y) & -P_{s} f(x)|=| \int_{0}^{1} \frac{\partial}{\partial r}\left(P_{\xi(r)} f(\gamma(r))\right) d r \mid \\
& \leq \int_{0}^{1}\left(\left|\nabla P_{\xi(r)} f\right|(\gamma(r)) d(x, y)+(t-s)\left|\mathscr{L} P_{\xi(r)} f\right|(\gamma(r))\right) d r \\
& \leq \int_{0}^{1} \sqrt{\mathrm{e}^{-2 K \xi(r)} d(x, y)^{2}+\frac{(t-s)^{2}(N+p-2) K}{\mathrm{e}^{2 K \xi(r)}-1}} P_{\xi(r)}\left(|\nabla f|^{p_{*}}\right)(\gamma(r))^{2 / p_{*}} d r .
\end{aligned}
$$

Now we are ready for approximation as a second step. Let $f$ be Lipschitz with a compact support. Then there is $f_{n} \in C_{0}^{\infty}(M)$ with $\sup _{n}\left\|\nabla f_{n}\right\|_{\infty}<\infty$ such that $f_{n} \rightarrow f$ pointwisely and $\left|\nabla f_{n}\right| \rightarrow|\nabla f|$ a.e. Since the last inequality holds for $f_{n}$, the same does for $f$. Then a usual truncation argument yields the same for $f \in C_{b}^{\mathrm{Lip}}(M)$. Then the conclusion follows by Proposition 3.2.

Lemma 4.5 (4.8) for each $x, y \in M$ and $\tau_{1}, \tau_{2}>0$ implies (2.10) and (2.11).

Proof. By virtue of Theorem 2.1, it suffices to show (2.11) for $f \in C_{b}^{\operatorname{Lip}}(M)$. By Proposition 3.1 and Proposition 3.2, (4.8) yields

$$
\left|\nabla P_{t} f\right|^{2} \leq \mathrm{e}^{-2 K t} P_{t}\left(|\nabla f|^{p_{*}}\right)^{2 / p_{*}}-\frac{4 K t^{2}}{(N+p-2)\left(1-\mathrm{e}^{-2 K t}\right)}\left(\mathscr{L} P_{t} f\right)^{2}
$$

for $f \in C_{b}^{\mathrm{Lip}}(M)$. Then, by applying Proposition 4.4 (ii) $\Rightarrow$ (i), we obtain (2.11) for $f \in C_{b}^{\mathrm{Lip}}(M)$.

Remark 4.6 It is tempting to study Proposition 4.4 in the framework of Bakry-Émery theory. For instance, if Proposition 4.4 (iii) follows directly from (2.9), then we can avoid the use of coupling method on which we are relying. However, the author do not know whether there is such a simpler way or not. Let us observe that a weaker estimate follows by an easy application of the Bochner-Weitzenböck formula:

$$
\Gamma_{2}(f)=\|\operatorname{Hess} f\|_{\mathrm{HS}}^{2}+\left(\operatorname{Ric}-(\nabla Z)^{b}\right)(\nabla f, \nabla f),
$$

where $\|\cdot\|_{\mathrm{HS}}$ stands for the Hilbert-Schmidt norm. Since we have

$$
\begin{aligned}
\frac{1}{N}(\mathscr{L} f)^{2} & \leq \frac{1}{m}(\Delta f)^{2}+\frac{1}{N-m}(Z f)^{2} \leq\|\operatorname{Hess} f\|_{\mathrm{HS}}^{2}+\frac{1}{N-m}(Z f)^{2}, \\
\left.\left.|\nabla| \nabla f\right|^{2}\right|^{2} & \leq 4\|\operatorname{Hess} f\|_{\mathrm{HS}}^{2}|\nabla f|^{2}
\end{aligned}
$$


one can show that (2.9) yields

$$
\begin{aligned}
& \left(|\nabla f|^{2}+\delta\right)\left(\Gamma_{2}(f)-K|\nabla f|^{2}-\frac{1}{N(p-1)}(\mathscr{L} f)^{2}\right) \geq\left.\left.\frac{p-2}{4(p-1)}|\nabla| \nabla f\right|^{2}\right|^{2}, \\
& \left(|\nabla f|^{2}+\delta\right)\left(\Gamma_{2}(f)-K|\nabla f|^{2}-\frac{1}{N+p-2}(\mathscr{L} f)^{2}\right) \geq\left.\left.\frac{p-2}{4(N+p-2)}|\nabla| \nabla f\right|^{2}\right|^{2},
\end{aligned}
$$

both of which are weaker than Proposition 4.4 (iii) (Recall $N \geq m \geq 1$ and $p \geq 2$ ).

We close this section with noting that Lemma 4.2, Lemma 4.3, Proposition 4.4 and Lemma 4.5 are all valid without the absence of the cut locus. Indeed, we will use them again in the next section (For Lemma 4.2, we will use a generalization of it).

\subsection{Coupling Method Via Discrete APproximation}

To make the argument in the last section rigorous even in the presence of the cut locus, we will approximate the coupling of diffusion processes by a sequence of couplings of geodesic random walks.

Let $\left(\gamma_{x y}\right)_{x, y \in M}$ be a family of unit-speed minimal geodesics defined on $[0, d(x, y)]$ such that $\gamma_{x y}$ goes from $x$ to $y$. By using a measurable selection theorem (e.g. [17, Theorem 6.9.6]), we will take $\gamma_{x y}$ as a measurable function of $(x, y)$ (more precisely, we will take a measurable choice of constant speed geodesics parametrized on $[0,1]$ and we take $\left(\gamma_{x y}\right)_{x, y}$ as their reparametrization). Without loss of generality, we may assume that $\gamma_{x y}$ is symmetric, that is, $\gamma_{x y}(d(x, y)-s)=\gamma_{y x}(s)$ holds. Similarly as in the last section, we denote the parallel transport along $\gamma_{x y}$ by $/ / x y$. We use the same symbol for parallel transport of orthonormal frames. Set $D(M):=\{(x, x) \mid x \in M\}$. Let $\Phi: M \rightarrow \mathscr{O}(M)$ be a measurable section of $\mathscr{O}(M)$. Let us define two measurable maps $\Phi_{i}: M \times M \rightarrow \mathscr{O}(M)$ for $i=1,2$ by

$$
\begin{aligned}
& \Phi_{1}(x, y):=\Phi(x), \\
& \Phi_{2}(x, y):= \begin{cases}/ / x y \Phi_{1}(x, y), & (x, y) \in M \times M \backslash D(M), \\
\Phi(x), & (x, y) \in D(M) .\end{cases}
\end{aligned}
$$

Let $\left(\zeta_{n}\right)_{n \in \mathbb{N}}$ be independent and identically distributed random variables whose distributions are uniform on the unit disk on $\mathbb{R}^{m}$. Take $x_{1}, x_{2} \in M$ and $\tau_{2}>\tau_{1}>0$. Set $t_{n}^{(k)}:=k^{-2} n$ for $k \in \mathbb{N}$ and $n \in \mathbb{N}_{0}$ with $n \leq k^{2}$. By using $\Phi_{i}$, we define a coupled geodesic random walk $\mathbf{X}^{k}(t)=\left(X_{1}^{k}(t), X_{2}^{k}(t)\right)$ with a discretization parameter $k \in \mathbb{N}$ by $X_{i}^{k}(0)=x_{i}$ and, for $t \in\left[t_{n}^{(k)}, t_{n+1}^{(k)}\right]$,

$$
\begin{aligned}
\tilde{\zeta}_{n+1}^{i} & :=\sqrt{2(m+2)} \Phi_{i}\left(\mathbf{X}^{k}\left(t_{n}^{(k)}\right)\right) \zeta_{n+1} \\
X_{i}^{k}(t) & :=\exp _{X_{i}^{k}\left(t_{n}^{(k)}\right)}\left(\sqrt{\tau_{i}} k^{2}\left(t-t_{n}^{(k)}\right)\left(k^{-1} \tilde{\zeta}_{n+1}^{i}+k^{-2} Z\right)\right)
\end{aligned}
$$

for $i=1,2$, where $\exp _{x}$ is the exponential map at $x$. By [26, Theorem 3.1] (see references therein also), $X_{i}^{k}(t)$ converges in law in $C([0, \infty) \rightarrow M)$ to an time-scaled $\mathscr{L}$-diffusion 
process with scale parameter $\tau_{i}$ starting from $x_{i}$ for $i=1,2$ respectively. Thus $\left(\mathbf{X}^{k}\right)_{k \in \mathbb{N}}$ is tight and hence a subsequential limit $\mathbf{X}^{k_{j}} \rightarrow \mathbf{X}=\left(X_{1}, X_{2}\right)$ in law in $C([0, \infty) \rightarrow M \times M)$ exists. Here "time-scaled by $\tau_{i}$ " means that the law of $X_{i}(\cdot)$ is the same as $\mathbb{P}_{x_{i}} \circ X\left(\tau_{i} \cdot\right)$. We fix such a subsequence $\left(k_{j}\right)_{j \in \mathbb{N}}$. In the rest of this paper, we use the same symbol $\mathbf{X}^{k}$ for the subsequence $\mathbf{X}^{k_{j}}$ and the term " $k \rightarrow \infty$ " always means the subsequential limit " $j \rightarrow \infty "$.

Set $\rho^{(k)}(n):=d\left(\mathbf{X}^{k}\left(t_{n}^{(k)}\right)\right)$. We first show a difference inequality for $\rho^{(k)}(n)$, which corresponds to the Itô formula (Lemma 4.7 below). To state it, we further introduce some notations. Let $\tilde{\zeta}_{n+1}^{\perp}(0)$ be the orthogonal projection of $\tilde{\zeta}_{n+1}$ to the hyperplane being perpendicular to $\dot{\gamma}_{\mathbf{X}^{k}\left(t_{n}^{(k)}\right)}(0)$. We denote a vector field along $\gamma_{\mathbf{X}^{k}\left(t_{n}^{(k)}\right)}$ given by parallel transport of $\tilde{\zeta}_{n+1}^{\perp}(0)$ by $\left(\tilde{\zeta}_{n+1}^{\perp}(s)\right)_{s \in\left[0, \rho^{(k)}(n)\right]}$ and we define $V_{n+1}(s):=\varphi_{\rho^{k}(n)}(s) \tilde{\zeta}_{n+1}^{\perp}(s)$, where $\varphi_{\rho^{k}(n)}$ was defined in (4.5). Take $v \in \mathbb{R}^{m}$ with $|v|=1$. Let us define $\lambda_{n+1}$ and $\Lambda_{n+1}$ by

$$
\begin{aligned}
\lambda_{n+1}: & := \begin{cases}\left\langle\tilde{\zeta}_{n+1}^{1}(0), \dot{\gamma}_{\mathbf{X}^{k}\left(t_{n}^{(k)}\right)}(0)\right\rangle & \text { if } \mathbf{X}^{k}\left(t_{n}^{(k)}\right) \notin D(M), \\
\sqrt{2(m+2)}\left\langle\zeta_{n+1}, v\right\rangle & \text { otherwise, }\end{cases} \\
\Lambda_{n+1}: & :\left(\left.\varphi_{\rho^{k}(n)}(s)\left\langle Z\left(t_{n}^{(k)}\right), \dot{\gamma}_{\mathbf{X}^{k}\left(t_{n}^{(k)}\right)}(s)\right\rangle\right|_{s=0} ^{\rho^{k}(n)}+\frac{1}{2} I_{\mathbf{X}^{k}\left(t_{n}^{(k)}\right)}\left(V_{n+1}, V_{n+1}\right)\right) 1_{\left\{\mathbf{X}^{k}\left(t_{n}^{(k)}\right) \notin D(M)\right\}},
\end{aligned}
$$

where $I_{z w}$ stands for the index form associated with $\gamma_{z w}$ as in Section 4.1. Fix a reference point $o \in M$. For $R>0$, let us define $\sigma_{R}: C([0, \infty) \rightarrow M \times M) \rightarrow[0, \infty]$ by $\sigma_{R}\left(w_{1}, w_{2}\right):=$ $\inf \left\{t \geq 0 \mid d\left(o, w_{1}(t)\right) \vee d\left(o, w_{2}(t)\right) \geq R\right\}$. By [26, Proposition 3.4], we have the following:

$$
\limsup _{R \rightarrow \infty} \limsup _{k \rightarrow \infty} \mathbb{P}\left[\sigma_{R}\left(\mathbf{X}^{k}\right)<\infty\right]=0 .
$$

We denote the discretization of $\sigma_{R}\left(\mathbf{X}^{k}\right)$ by $\hat{\sigma}_{R}$, that is,

$$
\hat{\sigma}_{R}:=\min \left\{n \in \mathbb{N} \mid t_{n-1}^{(k)}<\sigma_{R}\left(\mathbf{X}^{k}\right) \leq t_{n}^{(k)}\right\} .
$$

Lemma 4.7 Let $g \in C^{2}([0, \infty))$ be non-decreasing with $g^{\prime}(0)=0$ and $R>0$ sufficiently large. Then there exists $k_{0} \in \mathbb{N}$ such that, for any $k \geq k_{0}$,

$$
\begin{aligned}
g\left(\rho^{(k)}(n+1)\right) \leq & g\left(\rho^{(k)}(n)\right)+\frac{1}{k}\left(\sqrt{\tau_{2}}-\sqrt{\tau_{1}}\right) g^{\prime}\left(\rho^{(k)}(n)\right) \lambda_{n+1}+\frac{1}{k^{2}} g^{\prime}\left(\rho^{(k)}(n)\right) \Lambda_{n+1} \\
& +\frac{1}{2 k^{2}}\left(\sqrt{\tau_{2}}-\sqrt{\tau_{1}}\right)^{2} g^{\prime \prime}\left(\rho^{(k)}(n)\right) \lambda_{n+1}^{2}+\frac{1}{R k^{2}}
\end{aligned}
$$

holds on $\left\{n<\hat{\sigma}_{R}\right\} \cap\left\{\rho^{(k)}(n)>R^{-1}\right\}$.

Proof. When Cut $=\emptyset$, the assertion is an immediate consequence of the Taylor expansion, the first and second variational formulae and the index lemma. To take singularity at the cut locus into account, we will develop a more detailed argument based on the idea in [26, Lemma 4.4]. 
Let us define $H \subset M^{3}$ and $p_{1}, p_{2}: H \rightarrow M^{2}$ by

$$
\begin{aligned}
& H:=\left\{\begin{array}{l|l}
(x, y, z) \in M^{3} & \begin{array}{l}
x, y, z \in \overline{B(o, 3 R)}, \\
d(x, y) \geq R^{-1}, \\
d(x, y)=2 d(x, z)=2 d(y, z)
\end{array}
\end{array}\right\}, \\
& p_{1}(x, y, z):=(x, z), \\
& p_{2}(x, y, z):=(y, z) .
\end{aligned}
$$

If $\mathbf{q}=(x, y, z) \in H$, then $p_{1}(\mathbf{q}), p_{2}(\mathbf{q}) \notin$ Cut since $z$ is on a midpoint of a minimal geodesic joining $x$ and $y$. Since $H$ is compact, $p_{1}(H)$ and $p_{2}(H)$ are also compact. Hence both $p_{1}(H)$ and $p_{2}(H)$ are uniformly away from Cut $\cap \overline{B(o, 3 R)}$ since the cut locus is closed. Set

$$
\begin{aligned}
& z_{n}:=\gamma_{\mathbf{X}^{k}\left(t_{n}^{(k)}\right)}\left(\frac{\rho^{(k)}(n)}{2}\right) \\
& z_{n}^{\prime}:=\exp _{z_{n}}\left(V_{n+1}\left(\frac{\rho^{(k)}(n)}{2}\right)+\varphi_{\rho^{(k)}(n)}\left(\frac{\rho^{(k)}(n)}{2}\right) Z\right) .
\end{aligned}
$$

By the triangle inequality, we have

$$
\begin{gathered}
\rho^{(k)}(n)=d\left(X_{1}^{k}\left(t_{n}^{(k)}\right), z_{n}\right)+d\left(z_{n}, X_{2}^{k}\left(t_{n}^{(k)}\right)\right), \\
\rho^{(k)}(n+1) \leq d\left(X_{1}^{k}\left(t_{n+1}^{(k)}\right), z_{n}^{\prime}\right)+d\left(z_{n}^{\prime}, X_{2}^{k}\left(t_{n+1}^{(k)}\right)\right) .
\end{gathered}
$$

Let us denote the difference of the segmented distances by $\Theta_{1}$ and $\Theta_{2}$, that is,

$$
\begin{aligned}
& \Theta_{1}:=d\left(X_{1}^{k}\left(t_{n+1}^{(k)}\right), z_{n}^{\prime}\right)-d\left(X_{1}^{k}\left(t_{n}^{(k)}\right), z_{n}\right), \\
& \Theta_{2}:=d\left(z_{n}^{\prime}, X_{2}^{k}\left(t_{n+1}^{(k)}\right)\right)-d\left(z_{n}, X_{2}^{k}\left(t_{n}^{(k)}\right)\right) .
\end{aligned}
$$

Suppose $t_{n}^{(k)}<\sigma_{R}\left(\mathbf{X}^{k}\right)$ and $\rho^{(k)}(n) \geq R^{-1}$. Since $g$ is non-decreasing, we obtain

$$
\begin{aligned}
& g\left(\rho^{(k)}(n+1)\right)-g\left(\rho^{(k)}(n)\right) \\
& \quad \leq g\left(d\left(X_{1}^{k}\left(t_{n+1}^{(k)}\right), z_{n}^{\prime}\right)+d\left(z_{n}^{\prime}, X_{2}^{k}\left(t_{n+1}^{(k)}\right)\right)\right)-g\left(d\left(X_{1}^{k}\left(t_{n}^{(k)}\right), z_{n}\right)+d\left(z_{n}, X_{2}^{k}\left(t_{n}^{(k)}\right)\right)\right) \\
& \quad \leq g^{\prime}\left(\rho^{(k)}(n)\right)\left(\Theta_{1}+\Theta_{2}\right)+\frac{g^{\prime \prime}\left(\rho^{(k)}(n)\right)}{2}\left(\Theta_{1}+\Theta_{2}\right)^{2}+\frac{1}{R}\left(\Theta_{1}+\Theta_{2}\right)^{2}
\end{aligned}
$$

for sufficiently large $k$, uniformly in the position of $\mathbf{X}^{k}\left(t_{n}^{(k)}\right)$. Note that $\left(X_{1}^{k}\left(t_{n+1}^{(k)}\right), z_{n}^{\prime}\right)$ is uniformly away from the cut locus since $\left(\mathbf{X}^{k}\left(t_{n}^{(k)}\right), z_{n}\right) \in H$. Thus the first and second variational formulae yield that, by denoting the index form along the restriction of $\gamma_{\mathbf{X}^{k}\left(t_{n}^{(k)}\right)}$ to the geodesic from $X_{1}^{k}\left(t_{n}^{(k)}\right)$ to $z_{n}$ by $I_{1}$,

$$
\begin{aligned}
\mid \Theta_{1} & -\left.\frac{1}{k} \varphi_{\rho^{(k)}(n)}(s)\right|_{s=0} ^{\rho^{(k)}(n) / 2} \lambda_{n+1} \mid \leq \frac{1}{R k} \\
\Theta_{1} \leq & \left.\frac{1}{k} \varphi_{\rho^{k}(n)}(s)\right|_{s=0} ^{\rho^{(k)}(n) / 2} \lambda_{n+1} \\
& +\frac{1}{k^{2}}\left(\left.\varphi_{\rho^{(k)}(n)}(s)\left\langle Z, \dot{\gamma}_{\mathbf{X}^{k}\left(t_{n}^{(k)}\right)}\right\rangle_{\gamma_{\mathbf{X}^{k}\left(t_{n}^{(k)}\right)}(s)}\right|_{s=0} ^{\rho^{(k)}(n) / 2}+\frac{1}{2} I_{1}\left(V_{n+1}, V_{n+1}\right)\right) 1_{\left\{\mathbf{X}^{k}\left(t_{n}^{(k)}\right) \notin D(M)\right\}} \\
& +\frac{1}{R k^{2}}
\end{aligned}
$$


for sufficiently large $k$ uniformly in the position of $\mathbf{X}^{k}\left(t_{n}^{(k)}\right)$. In the same way, the corresponding estimate also holds true for $\Theta_{2}$. Then the assertion follows by plugging these estimates into (4.12).

The next lemma estimates the expectation of the second variation term in Lemma 4.7. We will use the convention $\sum_{n=i}^{i^{\prime}} b_{n}=0$ for any sequence $\left(b_{n}\right)_{n}$ when $i^{\prime}<i$.

Lemma 4.8 Let $n_{1}, n_{2} \in \mathbb{N}_{0}$ with $n_{1}<n_{2} \leq k^{2}$ and $R>0$ sufficiently large. Let $\mathcal{F}_{n}:=\sigma\left(\zeta_{1}, \ldots, \zeta_{n}\right)$.

(i) For each $h \in C([0, \infty) \rightarrow[0, \infty))$ and two $\mathcal{F}_{n}$-stopping times $S, T$ with $n_{1} \wedge \hat{\sigma}_{R} \leq$ $S \leq T \leq n_{2} \wedge \hat{\sigma}_{R}$

$$
\mathbb{E}\left[\sum_{i=S+1}^{T} h\left(\rho^{(k)}(i-1)\right) \Lambda_{i}\right] \leq \mathbb{E}\left[\sum_{i=S+1}^{T} h\left(\rho^{(k)}(i-1)\right) \Psi\left(\rho^{(k)}(i-1)\right)\right] .
$$

(ii) Let $g \in C^{2}([0, \infty))$ be non-decreasing, $g^{\prime}(0)=0$ and $g^{\prime \prime}(0) \geq 0$. Then there exists $k_{0} \in \mathbb{N}$ and $C>0$ being independent of $n_{1}, n_{2}$ and $R>1$ such that, for any $k \geq k_{0}$,

$$
\begin{aligned}
\mathbb{E} & {\left[g\left(\rho^{(k)}\left(n_{2} \wedge \hat{\sigma}_{R}\right)\right)\right] \leq \mathbb{E}\left[g\left(\rho^{(k)}\left(n_{1} \wedge \hat{\sigma}_{R}\right)\right)\right] } \\
& +\frac{1}{k^{2}} \mathbb{E}\left[\sum_{i=n_{1} \wedge \hat{\sigma}_{R}+1}^{n_{2} \wedge \hat{\sigma}_{R}} g^{\prime}\left(\rho^{(k)}(i-1)\right) \Psi\left(\rho^{(k)}(i-1)\right)+g^{\prime \prime}\left(\rho^{(k)}(i-1)\right)\left(\sqrt{\tau_{2}}-\sqrt{\tau_{1}}\right)^{2}\right] \\
& +\frac{C\left(n_{2}-n_{1}\right)}{R k^{2}} .
\end{aligned}
$$

Proof. (i) Let $\bar{\Lambda}_{n}:=\mathbb{E}\left[\Lambda_{n} 1_{\left\{n_{1} \wedge \hat{\sigma}_{R}<n \leq n_{2} \wedge \hat{\sigma}_{R}\right\}} \mid \mathcal{F}_{n-1}\right]$. Then we have

$$
\mathbb{E}\left[\sum_{i=S+1}^{T} h\left(\rho^{(k)}(i-1)\right) \Lambda_{i}\right]=\mathbb{E}\left[\sum_{i=S+1}^{T} h\left(\rho^{(k)}(i-1)\right) \bar{\Lambda}_{i}\right]
$$

since $\sum_{i=S \wedge n+1}^{T \wedge n} h\left(\rho^{(k)}(i-1)\right)\left(\Lambda_{i}-\bar{\Lambda}_{i}\right)$ is an $\mathcal{F}_{n}$-martingale. Let $\left(\hat{e}_{l}\right)_{l=1}^{m}$ be an orthonormal basis of $T_{X_{1}^{k}\left(t_{n}^{(k)}\right)} M$ with $\hat{e}_{1}=\dot{\gamma}_{\mathbf{X}^{k}\left(t_{n}^{(k)}\right)}(0)$ and $\hat{e}_{l}(s)$ a vector field along $\gamma_{\mathbf{X}^{k}\left(t_{n}^{(k)}\right)}$ given by parallel transport of $\hat{e}_{l}$. We also define a vector field $\bar{V}^{(l)}$ along $\gamma_{\mathbf{X}^{k}\left(t_{n}^{(k)}\right)}$ by $\bar{V}^{(l)}(s):=\varphi_{\rho^{(k)}(n)}(s) \hat{e}_{l}(s)$ for $l=2, \ldots, n$. For components of $\zeta_{n}=\left(\zeta_{n}^{(1)}, \ldots, \zeta_{n}^{(m)}\right)$, we have $\mathbb{E}\left[\zeta_{n}^{(l)} \zeta_{n}^{\left(l^{\prime}\right)}\right]=(m+2)^{-1} \delta_{l l^{\prime}}$. It yields

$$
\bar{\Lambda}_{n}=\left(\left.\varphi_{\rho^{(k)}(n)}(s)\left\langle Z, \dot{\gamma}_{\mathbf{X}^{k}\left(t_{n}^{(k)}\right)}\right\rangle_{\gamma_{\mathbf{X}^{k}\left(t_{n}^{(k)}\right)}(s)}\right|_{s=0} ^{\rho^{(k)}(n)}+\sum_{l=2}^{m} I_{\mathbf{X}^{k}\left(t_{n}^{(k)}\right)}\left(\bar{V}^{(l)}, \bar{V}^{(l)}\right)\right) 1_{\left\{\mathbf{X}^{k}\left(t_{n}^{(k)}\right) \notin D(M)\right\}}
$$

on $\left\{n_{1} \wedge \hat{\sigma}_{R}<n \leq n_{2} \wedge \hat{\sigma}_{R}\right\}$. Then, based on (2.9), a similar argument as [30, Lemma 3.4] yields

$$
\bar{\Lambda}_{n} \leq(N-1) \int_{0}^{\rho^{(k)}(n)} \varphi_{\rho^{(k)}(n)}^{\prime}(u)^{2} d u-K \int_{0}^{\rho^{(k)}(n)} \varphi_{\rho^{(k)}(n)}(u)^{2} d u
$$


on $\left\{n_{1} \wedge \hat{\sigma}_{R} n \leq n_{2} \wedge \hat{\sigma}_{R}\right\}$. Thus the conclusion holds in a similar way as in Lemma 4.2.

(ii) Let us define a sequence of $\mathcal{F}_{n}$-stopping times $S_{j}(j=0,1, \ldots)$ as follows:

$$
\begin{aligned}
S_{0} & :=n_{1} \wedge \hat{\sigma}_{R}, \\
S_{2 j+1} & :=\min \left\{n \geq S_{2 j} \mid \rho^{(k)}(n)<2 R^{-1}\right\} \wedge n_{2} \wedge \hat{\sigma}_{R}, \\
S_{2 j+2} & :=\min \left\{n>S_{2 j+1} \mid \rho^{(k)}(n)>\rho^{(k)}\left(S_{2 j+1}\right)\right\} \wedge n_{2} \wedge \hat{\sigma}_{R} .
\end{aligned}
$$

For simplicity of notations, set

$$
L(i):=g^{\prime}\left(\rho^{(k)}(i-1)\right) \Psi\left(\rho^{(k)}(i-1)\right)+g^{\prime \prime}\left(\rho^{(k)}(i-1)\right)\left(\sqrt{\tau_{2}}-\sqrt{\tau_{1}}\right)^{2} .
$$

Note that $\mathbb{E}\left[\lambda_{n+1} \mid \mathcal{F}_{n}\right]=0$ and $\mathbb{E}\left[\lambda_{n+1}^{2} \mid \mathcal{F}_{n}\right]=2$. Thus, as an immediate consequence of the first assertion and Lemma 4.7,

$$
\mathbb{E}\left[g\left(\rho^{(k)}\left(S_{2 j+1}\right)\right)\right] \leq \mathbb{E}\left[g\left(\rho^{(k)}\left(S_{2 j}\right)\right)\right]+\frac{1}{k^{2}} \mathbb{E}\left[\sum_{i=S_{2 j}+1}^{S_{2 j+1}} L(i)\right]+\frac{\mathbb{E}\left[S_{2 j+1}-S_{2 j}\right]}{R k^{2}}
$$

when $k$ is sufficiently large. On the other hand, since $\rho^{(k)}\left(S_{2 j+2}-1\right)>R^{-1}$ if $S_{2 j+1}<$ $n_{2} \wedge \hat{\sigma}_{R}$ and $k$ is sufficiently large,

$$
\begin{aligned}
\mathbb{E}\left[g\left(\rho^{(k)}\left(S_{2 j+2}\right)\right)\right] \leq \mathbb{E}[ & \left.g\left(\rho^{(k)}\left(S_{2 j+1}\right)\right)\right] \\
& +\mathbb{E}\left[\left(g\left(\rho^{(k)}\left(S_{2 j+2}\right)\right)-g\left(\rho^{(k)}\left(S_{2 j+2}-1\right)\right)\right) 1_{\left\{S_{2 j+1}<n_{2} \wedge \hat{\sigma}_{R}\right\}}\right] \\
\leq \mathbb{E}[ & \left.g\left(\rho^{(k)}\left(S_{2 j+1}\right)\right)\right] \\
& +\frac{1}{k^{2}} \mathbb{E}\left[L\left(S_{2 j+2}\right) 1_{\left\{S_{2 j+1}<n_{2} \wedge \hat{\sigma}_{R}\right\}}\right]+\frac{\mathbb{P}\left[S_{2 j+1}<n_{2} \wedge \hat{\sigma}_{R}\right]}{R k^{2}} .
\end{aligned}
$$

By using $g^{\prime}(0)=0$ and (4.6), we can show that there is a function $c:(0, \infty) \rightarrow \mathbb{R}$ with $\lim _{r \rightarrow 0} c(r)=0$ such that $g^{\prime}(r) \Psi(r) \geq c(r)$. This fact together with $g^{\prime \prime}(0) \geq 0$ implies that there is a constant $C>0$ such that

$$
0 \leq \frac{1}{k^{2}} \mathbb{E}\left[\sum_{i=S_{2 j+1}+1}^{S_{2 j+2}-1} L(i)\right]+\frac{C}{R k^{2}} \mathbb{E}\left[\left(S_{2 j+2}-S_{2 j+1}-1\right) \vee 0\right] .
$$

Note that $C$ can be chosen to be independent of $n_{1}$ and $n_{2}$. $C$ may depend on $R$ but it can be smaller for larger $R$. Thus we can choose it to be independent of $R$ also. Then the assertion holds by summing up (4.13), (4.14) and (4.15) and take a summation again with respect to $j$.

The third lemma deals with the limit $k \rightarrow \infty$ and a Gronwall type bound for expectations for truncated functions.

Lemma 4.9 Let $g$ be as in Lemma 4.8 (ii). Let $\psi \in C^{2}([0, \infty))$ be an increasing concave function satisfying $\psi(x)=x$ for $x \in[0,1]$ and $\psi(x)=2$ for $x \in[3, \infty)$ and set $\psi_{j}(x):=$ $j \psi(x / j)$ and $g_{j}:=g \circ \psi_{j}$ for $j \in \mathbb{N}$. Then, for $0 \leq s_{1}<s_{2} \leq 1$,

$$
\begin{aligned}
\mathbb{E}\left[g_{j}\left(d\left(\mathbf{X}\left(s_{2}\right)\right)\right)\right] \leq \mathbb{E}\left[g_{j}\left(d\left(\mathbf{X}\left(s_{1}\right)\right)\right)\right] & +\int_{s_{1}}^{s_{2}} \mathbb{E}\left[\left(g_{j}^{\prime} \cdot \Psi\right)(d(\mathbf{X}(u)))\right] d u \\
& +\left(\sqrt{\tau_{2}}-\sqrt{\tau_{1}}\right)^{2} \int_{s_{1}}^{s_{2}} \mathbb{E}\left[g_{j}^{\prime \prime}(d(\mathbf{X}(u)))\right] d u .
\end{aligned}
$$


Proof. Take $R>0$ sufficiently large. We set $\left\lfloor s_{i}\right\rfloor_{k}:=\inf \left\{n \in \mathbb{N} \mid t_{n}^{(k)}<s_{i} \leq t_{n+1}^{(k)}\right\}$. Note that $g_{j}, g_{j}^{\prime} \cdot \Psi$ and $g_{j}^{\prime \prime}$ are all uniformly continuous and bounded on $[0, \infty)$. Thus, by Lemma 4.8 (ii), for sufficiently large $k$, we have

$$
\begin{aligned}
& \mathbb{E}\left[g_{j}\left(d\left(\mathbf{X}^{k}\left(s_{2} \wedge \sigma_{R}\right)\right)\right)\right] \leq \mathbb{E}\left[g_{j}\left(d\left(\mathbf{X}^{k}\left(s_{1} \wedge \sigma_{R}\right)\right)\right)\right] \\
& +\frac{1}{k^{2}} \mathbb{E}\left[\sum_{i=\left\lfloor s_{1}\right\rfloor_{k} \wedge \hat{\sigma}_{R}+1}^{\left\lfloor s_{2}\right\rfloor_{k} \wedge \hat{\sigma}_{R}}\left(g_{j}^{\prime} \cdot \Psi\right)\left(d\left(\mathbf{X}^{k}\left(t_{i-1}^{(k)}\right)\right)\right)+g_{j}^{\prime \prime}\left(d\left(\mathbf{X}^{k}\left(t_{i-1}^{(k)}\right)\right)\right)\left(\sqrt{\tau_{2}}-\sqrt{\tau_{1}}\right)^{2}\right]+\frac{2}{R} \\
& \leq \mathbb{E}\left[g_{j}\left(d\left(\mathbf{X}^{k}\left(s_{1} \wedge \sigma_{R}\right)\right)\right)\right]+\mathbb{E}\left[\int_{s_{1} \wedge \sigma_{R}}^{s_{2} \wedge \sigma_{R}}\left(g_{j}^{\prime} \cdot \Psi\right)\left(d\left(\mathbf{X}^{k}(u)\right)\right) d u\right] \\
& \quad+\left(\sqrt{\tau_{2}}-\sqrt{\tau_{1}}\right)^{2} \mathbb{E}\left[\int_{s_{1} \wedge \sigma_{R}}^{s_{2} \wedge \sigma_{R}} g_{j}^{\prime \prime}\left(d\left(\mathbf{X}^{k}(u)\right)\right) d u\right]+\frac{3}{R} .
\end{aligned}
$$

Since $\left\{\mathbf{w} \mid \sigma_{R-1}(\mathbf{w})>s\right\}$ is open in $C([0, \infty) \rightarrow M \times M)$, the Portmanteau theorem for the weak convergence $\mathbf{X}^{k} \rightarrow \mathbf{X}$ yields

$$
\begin{aligned}
\liminf _{R \rightarrow \infty} \liminf _{k \rightarrow \infty} \mathbb{E}\left[g _ { j } \left(d \left(\mathbf { X } ^ { k } \left(s_{2}\right.\right.\right.\right. & \left.\left.\left.\left.\wedge \sigma_{R}\right)\right)\right)\right] \geq \liminf _{R \rightarrow \infty} \liminf _{k \rightarrow \infty} \mathbb{E}\left[g_{j}\left(d\left(\mathbf{X}^{k}\left(s_{2}\right)\right)\right) ; \sigma_{R}\left(\mathbf{X}^{k}\right)>s_{2}\right] \\
& \geq \liminf _{R \rightarrow \infty} \mathbb{E}\left[g_{j}\left(d\left(\mathbf{X}\left(s_{2}\right)\right)\right) ; \sigma_{R}(\mathbf{X})>s_{2}\right]=\mathbb{E}\left[g_{j}\left(d\left(\mathbf{X}\left(s_{2}\right)\right)\right)\right],
\end{aligned}
$$

where the last inequality follows from the fact that $X$ is conservative. On the other hand, for any $h \in C_{b}([0, \infty))$, (4.11) yields

$$
\begin{aligned}
& \limsup _{R \rightarrow \infty} \limsup _{k \rightarrow \infty} \mathbb{E}\left[\int_{s_{1} \wedge \sigma_{R}}^{s_{2} \wedge \sigma_{R}} h\left(d\left(\mathbf{X}^{k}(u)\right)\right) d u\right] \\
& \leq\|h\|_{\infty}\left(s_{2}-s_{1}\right) \limsup _{R \rightarrow \infty} \limsup _{k \rightarrow \infty} \mathbb{P}\left[\sigma_{R}\left(\mathbf{X}^{k}\right) \leq s_{2}\right] \\
& +\limsup _{R \rightarrow \infty} \limsup _{k \rightarrow \infty} \mathbb{E}\left[\int_{s_{1}}^{s_{2}} h\left(d\left(\mathbf{X}^{k}(u)\right)\right) d u ; \sigma_{R}\left(\mathbf{X}^{k}\right)>s_{2}\right] \\
& \leq \limsup _{k \rightarrow \infty} \mathbb{E}\left[\int_{s_{1}}^{s_{2}} h\left(d\left(\mathbf{X}^{k}(u)\right)\right) d u\right]=\int_{s_{1}}^{s_{2}} \mathbb{E}[h(d(\mathbf{X}(u)))] d u
\end{aligned}
$$

A similar argument also works for the first term in the right hand side of (4.16). Then the conclusion follows by applying these estimates to (4.16), when we take the limit $k \rightarrow \infty$ and $R \rightarrow \infty$ after it.

We are now ready to show the key assertion (4.8) in the last section.

Proposition 4.10 (4.8) holds.

Proof. Let $\psi_{j}$ be as in Lemma 4.9. Note first that we have $\psi_{j}^{\prime}(u) u \leq \psi_{j}(u)$ and $\psi_{j}^{\prime \prime}(u) \leq 0$ for each $u \geq 0$ since $\psi$ is concave and $\psi(0)=0$. In addition, $\psi_{j}$ and $\psi_{j}^{\prime}$ is non-decreasing in $j$ and we have $\lim _{j \rightarrow \infty} \psi_{j}(u)=u, \lim _{j \rightarrow \infty} \psi_{j}^{\prime}(u)=1$ and $\lim _{j \rightarrow \infty} \psi_{j}^{\prime \prime}(u)=0$ for each 
$u \geq 0$. By applying Lemma 4.9 with $g(u):=u^{p}$ together with Lemma 4.3 , we obtain

$$
\begin{aligned}
\mathbb{E}\left[\psi_{j}\left(d\left(\mathbf{X}\left(s_{2}\right)\right)\right)^{p}\right] \leq \mathbb{E}\left[\psi_{j}\left(d\left(\mathbf{X}\left(s_{1}\right)\right)\right)^{p}\right] \\
\quad-p K \tau^{*} \int_{s_{1}}^{s_{2}} \mathbb{E}\left[\psi_{j}(d(\mathbf{X}(u)))^{p-1} \psi_{j}^{\prime}(d(\mathbf{X}(u))) d(\mathbf{X}(u))\right] d u \\
\quad+p(N-1)\left(\sqrt{\tau_{2}}-\sqrt{\tau_{1}}\right)^{2} \int_{s_{1}}^{s_{2}} \mathbb{E}\left[\psi_{j}(d(\mathbf{X}(u)))^{p-1} \frac{\psi_{j}^{\prime}(d(\mathbf{X}(u)))}{d(\mathbf{X}(u))}\right] d u \\
\quad+p(p-1)\left(\sqrt{\tau_{2}}-\sqrt{\tau_{1}}\right)^{2} \int_{s_{1}}^{s_{2}} \mathbb{E}\left[\psi_{j}(d(\mathbf{X}(u)))^{p-2} \psi_{j}^{\prime}(d(\mathbf{X}(u)))^{2}\right] d u \\
+p\left(\sqrt{\tau_{2}}-\sqrt{\tau_{1}}\right)^{2} \int_{s_{1}}^{s_{2}} \mathbb{E}\left[\psi_{j}(d(\mathbf{X}(u)))^{p-1} \psi_{j}^{\prime \prime}(d(\mathbf{X}(u)))\right] d u
\end{aligned}
$$

By neglecting non-positive terms, trivial bounds $\psi_{j}(u) \leq u$ and $\psi_{j}^{\prime}(u) \leq 1$, properties of $\psi_{j}$ stated at the beginning and the Hölder inequality yields

$$
\begin{aligned}
\mathbb{E}\left[\psi_{j}\left(d\left(\mathbf{X}\left(s_{2}\right)\right)\right)^{p}\right] \leq \mathbb{E}\left[\psi_{j}\left(d\left(\mathbf{X}\left(s_{1}\right)\right)\right)^{p}\right]-p(K \wedge 0) \tau^{*} \int_{s_{1}}^{s_{2}} \mathbb{E}\left[\psi_{j}(d(\mathbf{X}(u)))^{p}\right] d u \\
+p(N+p-2)\left(\sqrt{\tau_{2}}-\sqrt{\tau_{1}}\right)^{2} \int_{s_{1}}^{s_{2}} \mathbb{E}\left[\psi_{j}(d(\mathbf{X}(u)))^{p-2}\right] d u \\
\leq \mathbb{E}\left[\psi_{j}\left(d\left(\mathbf{X}\left(s_{1}\right)\right)\right)^{p}\right]-p(K \wedge 0) \tau^{*} \int_{s_{1}}^{s_{2}} \mathbb{E}\left[\psi_{j}(d(\mathbf{X}(u)))^{p}\right] d u \\
+p(N+p-2)\left(\sqrt{\tau_{2}}-\sqrt{\tau_{1}}\right)^{2} \int_{s_{1}}^{s_{2}} \mathbb{E}\left[\psi_{j}(d(\mathbf{X}(u)))^{p}\right]^{1-2 / p} d u
\end{aligned}
$$

Set $a_{j}(s):=\mathbb{E}\left[\psi_{j}(d(\mathbf{X}(s)))^{p}\right]$. Then the last inequality implies that, for $\delta \in(0,1)$,

$$
\begin{aligned}
& \limsup _{h \rightarrow 0} \frac{\mathrm{e}^{2(K \wedge 0) \tau^{*}(s+h)}\left(a_{j}(s+h)+\delta\right)^{2 / p}-\mathrm{e}^{2(K \wedge 0) \tau^{*} s}\left(a_{j}(s)+\delta\right)^{2 / p}}{h} \\
& \leq 2(K \wedge 0) \tau^{*} \mathrm{e}^{2(K \wedge 0) \tau^{*} s}\left(a_{j}(s)+\delta\right)^{2 / p-1} \delta \\
& \quad+2(N+p-2)\left(\sqrt{\tau_{2}}-\sqrt{\tau_{1}}\right)^{2} \mathrm{e}^{2(K \wedge 0) \tau^{*} s}\left(a_{j}(s)+\delta\right)^{2 / p-1} a_{j}(s)^{1-2 / p} \\
& \leq 2(N+p-2)\left(\sqrt{\tau_{2}}-\sqrt{\tau_{1}}\right)^{2} \mathrm{e}^{2(K \wedge 0) \tau^{*} s} .
\end{aligned}
$$

Thus the Gronwall lemma yields that there is a constant $C_{1}>0$ being independent of $j, s$ and $\delta$ such that $a_{j}(s) \leq C_{1}$ holds. Therefore the monotone limit $a_{\infty}:=\lim _{j \rightarrow \infty} a_{j}(s)$ exists in $\mathbb{R}$. In addition, the monotone convergence theorem yields $a_{\infty}(s)=\mathbb{E}\left[d(\mathbf{X}(s))^{p}\right]$.

With the aid of the monotone convergence theorem and the dominated convergence theorem, by letting $j \rightarrow \infty$ in (4.17) and by applying the Hölder inequality, we obtain

$$
a_{\infty}\left(s_{2}\right) \leq a_{\infty}\left(s_{1}\right)-p K \tau^{*} \int_{s_{1}}^{s_{2}} a_{\infty}(u) d u+p(N+p-2)\left(\sqrt{\tau_{2}}-\sqrt{\tau_{1}}\right)^{2} \int_{s_{1}}^{s_{2}} a_{\infty}(u)^{1-2 / p} d u
$$

Then we argue as in (4.18) with this inequality to apply the Gronwall inequality. Conse- 
quently, we obtain

$$
\begin{aligned}
\left(a_{\infty}(1)+\delta\right)^{2 / p} \leq \mathrm{e}^{-2 K \tau^{*}}\left(a_{\infty}(0)+\delta\right)^{2 / p}+2(N+p-2)\left(\sqrt{\tau_{2}}-\sqrt{\tau_{1}}\right)^{2} \mathrm{e}^{-2 K \tau^{*}} \int_{0}^{1} \mathrm{e}^{2 K \tau^{*} u} d u \\
+2(K \vee 0) \mathrm{e}^{-2 K \tau^{*}} \int_{0}^{1} \mathrm{e}^{2 K \tau^{*} u} d u \delta^{2 / p}
\end{aligned}
$$

Since $a_{\infty}(0)=d(x, y)^{p}$ and the definition of the Wasserstein distance and $\mathbf{X}$ implies $a_{\infty}(1) \geq W_{p}\left(P_{\tau_{1}}^{*} \delta_{x}, P_{\tau_{2}}^{*} \delta_{y}\right)^{p}$, the conclusion holds by letting $\delta \downarrow 0$ in the last inequality.

Proof of Theorem 2.6. The combination of Proposition 4.10 and Lemma 4.5 immediately completes the proof.

For $c: M \times M \rightarrow \mathbb{R}$ measurable and bounded from below, and $\mu_{1}, \mu_{2} \in \mathscr{P}(M)$, we define the optimal transportation cost $\mathcal{T}_{c}\left(\mu_{1}, \mu_{2}\right)$ between $\mu_{1}$ and $\mu_{2}$ associated with the cost function $c$ as follows:

$$
\mathcal{T}_{c}\left(\mu_{1}, \mu_{2}\right)=\inf \left\{\int_{M \times M} c d \pi \mid \pi \text { is a coupling of } \mu_{1} \text { and } \mu_{2}\right\} .
$$

As a variant of Proposition 4.10, we obtain the following:

Theorem 4.11 For $\tau_{1}, \tau_{2}>0, \mu_{1}, \mu_{2} \in \mathscr{P}(M)$ and $p \geq 2$,

$$
\mathcal{T}_{\mathfrak{s}_{K^{*}}^{p}(d / 2)}\left(P_{\tau_{1}}^{*} \mu_{1}, P_{\tau_{2}}^{*} \mu_{2}\right)^{2 / p} \leq \mathrm{e}^{-\theta} \mathcal{T}_{\mathfrak{s}_{K^{*}}^{p}(d / 2)}\left(\mu_{1}, \mu_{2}\right)^{2 / p}+\frac{(N+p-2)\left(1-\mathrm{e}^{-\theta}\right)}{2 \theta}\left(\sqrt{\tau_{2}}-\sqrt{\tau_{1}}\right)^{2},
$$

where $\theta=\theta\left(\tau_{1}, \tau_{2}, K, N, p\right):=K\left(\tau_{1}+\tau_{2}\right)+p K^{*}\left(\sqrt{\tau_{2}}-\sqrt{\tau_{1}}\right)^{2} / 2$, and $K^{*}=K /(N-1)$ as in the definition of $\Psi$ in (4.4).

Before entering the proof, we recall the following elementary relations for comparison functions:

$$
\begin{aligned}
& \mathfrak{s}_{K^{*}}^{\prime}=\mathfrak{c}_{K^{*}}, \quad \mathfrak{c}_{K^{*}}^{\prime}=-K^{*} \mathfrak{s}_{K^{*}}, \quad \mathfrak{c}_{K^{*}}^{2}+K^{*} \mathfrak{s}_{K^{*}}^{2}=1, \\
& \mathfrak{s}_{K^{*}}(2 r)=2 \mathfrak{s}_{K^{*}}(r) \mathfrak{c}_{K^{*}}(r), \quad \mathfrak{c}_{K^{*}}(2 r)=\mathfrak{c}_{K^{*}}(r)^{2}-K^{*} \mathfrak{s}_{K^{*}}(r)^{2} .
\end{aligned}
$$

Proof. The same argument as in Lemma 3.4 works for the transportation cost $\mathcal{T}_{\mathfrak{s}_{K^{*}}^{p}(d / 2)}$ instead of $W_{p}^{p}$. Hence it suffices to show the assertion only when both $\mu_{1}$ and $\mu_{2}$ are Dirac measures. We consider only the case $K \neq 0$ since the assertion is reduced to (4.8) when $K=0$. We begin with the integrability of $\mathfrak{s}_{K^{*}}(d(\mathbf{X}(s)))^{p}$ as in the proof of Proposition 4.10. Since it is obvious when $K>0$, we assume $K<0$ for a while. By 
applying Lemma 4.9 with $g(u)=\mathfrak{s}_{K^{*}}(u / 2)^{p}$ and (4.7) yield

$$
\begin{aligned}
\mathbb{E} & {\left[\mathfrak{s}_{K^{*}}\left(\frac{\psi_{j}\left(d\left(\mathbf{X}\left(s_{2}\right)\right)\right)}{2}\right)^{p}\right] \leq \mathbb{E}\left[\mathfrak{s}_{K^{*}}\left(\frac{\psi_{j}\left(d\left(\mathbf{X}\left(s_{1}\right)\right)\right)}{2}\right)^{p}\right] } \\
& -\frac{p K\left(\tau_{1}+\tau_{2}\right)}{2} \int_{s_{1}}^{s_{2}} \mathbb{E}\left[\left(\mathfrak{s}_{K^{*}}^{p-1} \cdot \mathfrak{c}_{K^{*}}\right)\left(\frac{\psi_{j}(d(\mathbf{X}(u)))}{2}\right) \psi_{j}^{\prime}(d(\mathbf{X}(u))) \mathfrak{t}_{K^{*}}\left(\frac{d(\mathbf{X}(u))}{2}\right)\right] d u \\
& +\frac{p(N-1)\left(\sqrt{\tau_{2}}-\sqrt{\tau_{1}}\right)^{2}}{2} \int_{s_{1}}^{s_{2}} \mathbb{E}\left[\left(\mathfrak{s}_{K^{*}}^{p-1} \cdot \mathfrak{c}_{K^{*}}\right)\left(\frac{\psi_{j}(d(\mathbf{X}(u)))}{2}\right) \frac{\psi_{j}^{\prime}(d(\mathbf{X}(u)))}{\mathfrak{s}_{K^{*}}(d(\mathbf{X}(u)))}\right] d u \\
& +\frac{p\left(\sqrt{\tau_{2}}-\sqrt{\tau_{1}}\right)^{2}}{4} \int_{s_{1}}^{s_{2}} \mathbb{E}\left[\left((p-1) \mathfrak{s}_{K^{*}}^{p-2}-p K^{*} \mathfrak{s}_{K^{*}}^{p}\right)\left(\frac{\psi_{j}(d(\mathbf{X}(u)))}{2}\right) \psi_{j}^{\prime}(d(\mathbf{X}(u)))^{2}\right] d u \\
& +\frac{p\left(\sqrt{\tau_{2}}-\sqrt{\tau_{1}}\right)^{2}}{2} \int_{s_{1}}^{s_{2}} \mathbb{E}\left[\left(\mathfrak{s}_{K^{*}}^{p-1} \cdot \mathfrak{c}_{K^{*}}\right)\left(\frac{\psi_{j}(d(\mathbf{X}(u)))}{2}\right) \psi_{j}^{\prime \prime}(d(\mathbf{X}(u)))\right] d u .
\end{aligned}
$$

Note that there exists $c_{j} \geq 1$ with $\lim _{j \rightarrow \infty} c_{j}=1$ such that $\mathfrak{c}_{K^{*}}\left(\psi_{j}(u) / 2\right) \cdot \mathfrak{t}_{K^{*}}(u / 2) \leq$ $c_{j} \mathfrak{s}_{K^{*}}\left(\psi_{j}(u) / 2\right)$ for each $u \geq 0$. Set $\tilde{a}_{j}(s):=\mathbb{E}\left[\mathfrak{s}_{K^{*}}\left(\psi_{j}(d(\mathbf{X}(s))) / 2\right)^{p}\right]$. By a similar argument as in the proof of Proposition 4.10, from (4.19), we obtain

$$
\tilde{a}_{j}\left(s_{2}\right) \leq \tilde{a}_{j}\left(s_{1}\right)-\frac{c_{j} p \theta}{2} \int_{s_{1}}^{s_{2}} \tilde{a}_{j}(u) d u+\frac{p(N+p-2)\left(\sqrt{\tau_{2}}-\sqrt{\tau_{1}}\right)^{2}}{4} \int_{s_{1}}^{s_{2}} \tilde{a}_{j}(u)^{1-2 / p} d u .
$$

Thus, as we discussed in (4.18), we can show that there exists $\tilde{C}_{1}>0$ being independent of $s$ and $j$ such that $\tilde{a}_{j}(s) \leq \tilde{C}_{1}$. It ensures $\tilde{a}_{\infty}(s):=\lim _{j \rightarrow \infty} \tilde{a}_{j}(s)<\infty$ for each $s \geq 0$.

Now we turn to the general situation $K \in \mathbb{R}$. (4.19) is still valid in this case. Then, by taking the limit $j \rightarrow \infty$, the conclusion follows in the same way as in the proof of Proposition 4.10.

\section{REFERENCES}

[1] L. Ambrosio, N. Gigli, A. Mondino, and T. Rajala, Riemannian Ricci curvature lower bounds in metric measure spaces with $\sigma$-finite measure, To appear in Trans. Amer. Math. Soc., 2012.

[2] L. Ambrosio, N. Gigli, and G. Savaré, Bakry-Émery curvature-dimension condition and Riemannian Ricci curvature bounds, Preprint. Available at: arXiv: 1209.5786.

$[3]$ , Calculus and heat flow in metric measure spaces and applications to spaces with Ricci bounds from below, To appear in Invent. Math. Available at: arXiv:1106.2090.

[4] _ Metric measure spaces with Riemannian Ricci curvature bounded from below, To appear in Duke Math. J. Available at: arXiv:1109.0222.

[5] _ Gradient flows in metric spaces and in the space of probability measures, second ed., Birkhäuser Verlag, Basel, 2008.

[6] _ Density of Lipschitz functions and equivalence of weak gradients in metric measure spaces, Rev. Mat. Iberoam. 29 (2013), no. 3, 969-996. 
[7] M. Arnaudon, K.A. Coulibaly, and A. Thalmaier, Horizontal diffusion in $C^{1}$ path space, Séminaire de Probabilités, XLIII, Lecture Notes in Mathematics, 2006, Springer, Berlin, 2011, pp. 73-94.

[8] K. Bacher and K.-T. Sturm, Localization and tensorization properties of the curvature-dimension condition for metric measure spaces, J. Funct. Anal. 259 (2010), no. $1,28-56$.

[9] D. Bakry, On Sobolev and logarithmic Sobolev inequalities for Markov semigroups, New trends in stochastic analysis (Charingworth, 1994), World Sci. Publ. River Edge, NJ, 1997, pp. 43-75.

[10] _ Functional inequalities for Markov semigroups, Probability measures on groups: recent directions and trends (Mumbai), Tata Inst. Fund. Res., 2006, pp. 91147.

[11] D. Bakry and M. Émery, Diffusions hypercontractives, Séminaire de probabilités, XIX, 1983/1984, Lecture notes in Mathematics, 1123, Springer, Berlin, 1985, pp. 177206.

[12] D. Bakry, I. Gentil, and M. Ledoux, Analysis and geometry of Markov diffusion operators Grundlehren der Mathematischen Wissenschaften, vol. 348, Springer-Verlag, 2014 .

[13] D. Bakry, I. Gentil, and M. Ledoux, On Harnack inequalities and optimal transport, To appear in Ann. Sc. Norm. Super. Pisa. Available at: arXiv:1210.4650.

[14] D. Bakry and M. Ledoux Sobolev inequalities and Myers's diameter theorem for an abstract Markov generator, Duke Math. J., 85 (2006) 253270.

[15] D. Bakry and M. Ledoux, A logarithmic Sobolev form of the Li-Yau parabolic inequality, Rev. Mat. Iberoam. 22 (2006), no. 2, 683-702.

[16] Z.M. Balogh, A. Engoulatov, L. Hunziker, and O.E. Maasalo, Functional inequalities and Hamilton-Jacobi equations in geodesic spaces, Potential Anal. 36 (2012), no. 2, 317-337.

[17] V. I. Bogachev, Measure theory. Vol. I, II Springer, Berlin, 2007.

[18] F. Bolley, I. Gentil, and A. Guillin, Dimensional contraction via Markov transportation distance, To appear in J. Lond. Math. Soc. Available at: arXiv:1304.1929.

[19] M. Cranston, Gradient estimates on manifolds using coupling, J. Funct. Anal. 99 (1991), no. 1, 110-124.

[20] M. Erbar, K. Kuwada, and K.-T. Sturm, On the equivalence of the entropic curvaturedimension condition and Bochner's inequality on metric measure spaces, Preprint. Available at: arXiv:1303.4382. 
[21] N. Gozlan, C. Roberto, and P.-M. Samson, Hamilton-jacobi equations on metric spaces and transport entropy inequalities, To appear in Rev. Mat. Iberoam. Available at: arXiv:1203.2783.

[22] E. P. Hsu, Stochastic analysis on manifolds, Graduate studies in mathematics, 38, American mathematical society, Providence, RI, 2002.

[23] W. Kendall, Nonnegative Ricci curvature and the Brownian coupling property, Stochastics 19 (1986), 111-129.

[24] K. Kuwada, Couplings of the Brownian motion via discrete apporoximation under lower Ricci curvature bounds, Probabilistic Approach to Geometry, Adv. Stud. Pure Math. 57, Math. Soc. Japan, 2010, pp. 273-292.

[25] _ Duality on gradient estimates and Wasserstein controls, J. Funct. Anal. 258 (2010), no. 11, 3758-3774.

[26] _ Convergence of time-inhomogeneous geodesic random walks and its application to coupling methods, Ann. Probab. 40 (2012), no. 5, 1945-1979.

[27] _ A probabilistic approach to the maximal diameter theorem, Math. Nachr. 286 (2013), no. 4, 374-378.

[28] _ Gradient estimate for Markov kernels, Wasserstein control and Hopf-Lax formula, Potential Theory and Related Fields, RIMS Kôkyûroku Bessatsu. B43 (2013) 61-80.

[29] K. Kuwada and R. Philipowski, Coupling of Brownian motion and Perelman's $\mathcal{L}$ functional, J. Funct. Anal. 260 (2011), no. 9, 2742-2766.

[30] K. Kuwada and K.-T. Sturm, Monotonicity of time-dependent transportation costs and coupling by reflection, Potential Anal. 39 (2013), no. 3, 231-263.

[31] M. Ledoux, The geometry of Markov diffusion generators, Ann. Fac. Sci. Toulouse Math. (6) 9 (2000), no. 2, 305-366.

[32] S. Lisini, Characterization of absolutely continuous curves in Wasserstein spaces, Calc. Var. Partial Differential Equations 28 (2007), no. 1, 85-120.

[33] J. Lott and C. Villani, Ricci curvature for metric-measure spaces via optimal transport, Ann. Math. 169 (2009), no. 3, 903-991.

[34] R. W. Neel and I. Popescu, A stochastic target approach to Ricci flow on surfaces, Preprint. Available at: arXiv:1209.4150.

[35] Z.-M. Qian, On conservation of probability and the Feller property, Ann. Prob. 24 (1996), no. 1, 280-292.

[36] T. Rajala, Interpolated measures with bounded density in metric spaces satisfying the curvature-dimension conditions of Sturm, J. Funct. Anal. 263 (2012), no. 4, 896-924. 
[37] G. Savaré, Self-improvement of the Bakry-Émery condition and Wasserstein contraction of the heat flow in $R C D(K, \infty)$ metric measure spaces, Disc. Cont. Dyn. Sist. 34 (2014), no. 4, 1641-1661.

[38] K.-T. Sturm, On the geometry of metric measure spaces. I, Acta. Math. 196 (2006), no. $1,65-131$.

[39] _ On the geometry of metric measure spaces. II, Acta. Math. 196 (2006), no. 1, $133-177$.

[40] P. Topping, L-optimal transportation for Ricci flow, J. Reine Angew. Math. 636 (2009), 93-122.

[41] C. Villani, Optimal transport, old and new, Grundlehren der Mathematischen Wissenschaften, vol. 338, Springer-Verlag, 2008.

[42] M.-K. von Renesse, Intrinsic coupling on Riemannian manifolds and polyhedra, Electron. J. Probab. 9 (2004), no. 14, 411-435.

[43] F.-Y. Wang, Functional inequalities, Markov semigroups, and spectral theory, Mathematics Monograph Series 4, Science Press, Beijing, China, 2005.

[44] _ Equivalent semigroup properties for curvature-dimension condition, Bull. Sci. Math. 135 (2011), no. 6-7, 803-815.

[45] _ Analysis for diffusion processes on Riemannian manifolds, World Scientific Publishing Co. Pte. Ltd., Hackensack, NJ, 2014. 